\title{
Solar $\mathrm{H} \alpha$ features with hot onsets
}

\section{Network fibrils ${ }^{\star}$}

\author{
Robert J. Rutten ${ }^{1,2,3}$, Luc H. M. Rouppe van der Voort ${ }^{2,3}$, and Bart De Pontieu ${ }^{4,2,3}$ \\ ${ }^{1}$ Lingezicht Astrophysics, 't Oosteneind 9, 4158 CA Deil, The Netherlands \\ e-mail: R.J.Rutten@uu.nl \\ ${ }^{2}$ Institute of Theoretical Astrophysics, University of Oslo, PO Box 1029, Blindern 0315, Oslo, Norway \\ 3 Rosseland Centre for Solar Physics, University of Oslo, PO Box 1029, Blindern 0315, Oslo, Norway \\ ${ }^{4}$ Lockheed-Martin Solar and Astrophysics Laboratory, 3251 Hanover Street, Palo Alto, CA 94304, USA \\ Received 16 June 2019 / Accepted 19 August 2019
}

\begin{abstract}
Even in quiet areas underneath coronal holes the solar chromosphere contains ubiquitous heating events. They tend to be small scale and short lived, hence difficult to identify. Here we do not address their much-debated contribution to outer-atmosphere heating, but their aftermaths. We performed a statistical analysis of high-resolution observations in the Balmer $\mathrm{H} \alpha$ line to suggest that many slender dark $\mathrm{H} \alpha$ fibrils spreading out from network represent cooling gas that outlines tracks of preceding rapid type II spicule events or smaller similar but as yet unresolved heating agents in which the main gas constituent, hydrogen, ionizes at least partially. Subsequent recombination then causes dark $\mathrm{H} \alpha$ fibrils enhanced by nonequilibrium overopacity. We suggest that the extraordinary fibrilar appearance of the $\mathrm{H} \alpha$ chromosphere around network results from intermittent, frequent small-scale prior heating.
\end{abstract}

Key words. Sun: chromosphere - Sun: magnetic fields

\section{Introduction}

The solar chromosphere is finely structured. Lockyer (1868) gave it its name for the colorful appearance of the prominence lines he saw rimming the entire Sun off-limb, primarily H I Balmer and He I lines (he also detected and named helium). Solar imaging in Balmer $\mathrm{H} \alpha$ shows that the on-disk counterpart of Lockyer's chromosphere consists of dense canopies of slender fibrils where there is some magnetic activity (e.g., Bray \& Loughhead 1974) and that these fibrils are highly variable with time. Thus, the solar chromosphere is a dynamically fibril-structured envelope around the thinner photospheric shell. In the meantime it became clear that it is the domain where hydrogen ionizes and where coronal heating and mass loading are rooted.

So-called type II spicules are an important chromospheric ingredient in "quiet" areas outside active regions. Since their first identification by De Pontieu etal. (2007a), they have attracted much attention and debate regarding their nature, their physical drivers, and their role as quiet-Sun sources of mass and energy loading of the corona. We refer to the recent studies of De Pontieu et al. (2017), Antolin et al. (2018), Martínez-Sykora et al. (2018), and Chintzoglou et al. (2018) for a current overview and references, but here we do not discuss these aspects further because we instead address another topic: their chromospheric aftermaths.

The analysis presented here targets $\mathrm{H} \alpha$ fibrils around moderately active network bordering coronal holes. It is a sequel to Rouppe van der Voort et al. (2009; henceforth Pub A) and Rutten \& Rouppe van der Voort (2017; henceforth Pub B) and uses the same $\mathrm{H} \alpha$ imaging sequences, both obtained with

\footnotetext{
$\star$ Movies associated to Fig. 3 and blinkers are available at https://www . aanda.org
}

the CRisp Imaging SpectroPolarimeter (CRISP, Scharmer et al. 2008) at the Swedish 1-m Solar Telescope (SST, Scharmer et al. 2003). The first study established that "rapid blue-shifted excursion" (RBE) fibrils in the outer blue wing of $\mathrm{H} \alpha$ are on-disk manifestations of off-limb type II spicules similar to those that were found before in Ca II $8542 \AA$ by Langangen et al. (2008). The same was then found for "rapid red-shifted excursion" (RRE) fibrils in the outer red wing of $\mathrm{H} \alpha$ by Sekse et al. (2013a). The introduction to Pub B reviewed these and also magnetoacoustic field-guided "dynamic fibrils" emanating fairly upright from plage and network, similar but shorter ones in sunspot chromospheres, and "long fibrils", which appear to span from magnetic roots in network or plage far out over adjacent internetwork cells. We refer to this summary rather than repeating it here.

Very thin long fibrils called "slender CaIIH fibrils" observed in the cores of the Ca II H\&K lines were studied by Jafarzadeh et al. (2017) after being reported earlier by Pietarila et al. (2009). These may also be on-disk representations of the type II spicule phenomenon, as are the comparable long, thin Ca II H "straws" close to the limb described by Rutten (2006), but this has not yet been verified.

This study of spicule II aftermaths is inspired by the example case in Pub B of a striking long, thick, dark $\mathrm{H} \alpha$ fibril that appeared minutes after a hot disturbance was launched from a small patch of moderately active network that was called a "propagating heating event" (PHE). It appeared similar to an RBE marking the launch of a type II spicule but extended unusually far. It was recognized in the far blue $\mathrm{H} \alpha$ wing from RBE-like combination of large blueshift and large broadening and became visible also in $304,171,193$, and $94 \AA$ images from the Atmospheric Imaging Assembly (AIA) on board the Solar Dynamics Observatory (SDO) as an accelerating PHE, an extending thin 
long jet-like feature that reached high temperatures, implying hydrogen ionization along its trajectory. Subsequently, minutes later, a dark $\mathrm{H} \alpha$ fibril appeared that outlined the PHE trajectory rather like the contrails marking preceding passages of airplanes on our sky. The dark fibril then retracted back to the original launch site with a conspicuous $\mathrm{H} \alpha$ core redshift. This Pub B case exemplified a heating event reaching hydrogen ionization along its track, followed by a return aftermath of back-flowing cooling gas with hydrogen recombination.

Naturally, the Pub B case led to the suspicion that similar but less fierce type II spicule launches manifested by RBEs and RREs may be important contributors to the fibrilar scenes that are so emphatically present in $\mathrm{H} \alpha$ images, in this case, long fibrils around network. Pub B reported searches for more such occurrences. A few more cases were indeed detected and reported, also at nearly the same location, which suggests recurrence, but the conclusion was that the fibrilar $\mathrm{H} \alpha$ scenes are too dynamic, too small scale, and too confused for easy one-to-one identification of line-core fibrils appearing a few minutes after blue-wing RBEs. The impression was that the slenderest fibrilar features showed best correspondence, but the issue was postponed to future analysis. We take it up here.

Thus, our questions are whether the appearance of contrail fibrils and return aftermath fibrils after similar PHE launches are common, and in particular, whether RBEs and RREs represent such launches. Regardless of the issue whether these chromospheric phenomena contribute significant heating and mass loading to the outer atmosphere, they do represent PHEs because they extend accelerating away from network at increasing temperature (e.g., Fig. 1 of Sekse et al. 2013b), with corresponding type II spicule tips often hotter than $20000 \mathrm{~K}$ and sometimes reaching million-degree temperatures (De Pontieu et al. 2011, see also Henriques et al. 2016).

If they indeed are followed by dark $\mathrm{H} \alpha$ contrail fibrils, then the latter constitute a category not present in the numerical $\mathrm{H} \alpha$ studies of Leenaarts et al. (2012, 2015), whose Bifrost simulation (Carlsson et al. 2016) does contain $\mathrm{H} \alpha$ fibrils connecting two opposite-polarity network patches but few fibrils emanating away from the network, and indeed does not contain RBEs either. Because the latter are ubiquitous around network, with only half-minute lifetimes but a recurrency rate of $0.7 \mathrm{~min}$ per location where they occur (Sekse et al. 2013b), RBE- and RREproduced fibrils may contribute much to the fibrilar appearance of the $\mathrm{H} \alpha$ chromosphere around network and represent a direct consequence of and companion to small-scale dynamic heating in the chromosphere outside active regions. Our results below suggest that this is the case.

We use the same data as in Pub B, but proceed from its singlecase study to full-field and full-duration analysis. In addition, we use the Pub A $\mathrm{H} \alpha$ data with which Rouppe van der Voort et al. (2009) established H $\alpha$ RBEs as type II spicule manifestations. Both data sets are suited because they combine high resolution with good wavelength coverage in $\mathrm{H} \alpha$ at high cadence while targeting isolated network patches near disk center that fit within the small SST field of view.

The next section summarizes the observations and our tools. Section 3 presents and describes figures that represent the core of this study. We interpret them in Sect. 4 and then add discussion and our conclusion.

\section{Observations and methods}

Data $A$ and $B$. We used the $\mathrm{H} \alpha$ spectral imaging sequences of Pub A and Pub B and call them data A and data B, respec- tively. Both were collected with the CRISP at the SST, and both targeted active network in a very quiet area near disk center that bordered a coronal hole (Fig. 1). In data B it contained a small pore that was present hours before but nearly vanished meanwhile. The observing durations were 24 and $72 \mathrm{~min}$ at spectralscan cadences $6.7 \mathrm{~s}$ and $11.5 \mathrm{~s}$. We refer to Pub A and Pub B for further detail on these observations and their reduction. The other data reported there are not used here.

In Pub A an automated detection procedure was used to identify $608 \mathrm{RBE}$ features in the 212 spectral image scans of the data $\mathrm{A} \mathrm{H} \alpha$ sequence. Each detection represented a single-image snapshot part of an RBE, including multiple detections of the same RBE because their lifetimes were often longer than the $6.7 \mathrm{~s}$ imaging cadence (up to a minute, histogram in Fig. 13 of Pub A). For each detection the skeleton of the RBE feature was determined and stored; this skeleton list is also used as input here.

Strous-format scatter correlation. In many figures we show pixel-by-pixel scatter relations between two image quantities in an informative format based on Figs. 13.2-13.3 of Strous (1994) and named after him. It is here obtained with IDL programs by A. G. and H. W. de Wijn (available online ${ }^{1}$ under IDL/cubelib). In this format, sample density contours are shown instead of individual symbols per pixel-pair sample for two reasons. The first is that when plotting many samples, these overlap and saturate into non-informative solid black. Contours then represent a better rendering of their joint probability density. The second reason is that plotting pixel-pair samples with a linear fit quantifying the Pearson correlation coefficient defined for linear dependence in the presence of noise is less applicable to typical solar scenes in which multiple and diverse agents cause varying responses, including nonlinearities. Plotting sample contours then permits recognizing characteristic behavior of particular agents and features, also rare ones, rather like interpreting mountain relief using elevation contours on a topographical map from which we glean some terms.

The first such figure (Fig. 5) shows the format for easyto-interpret agents and serves as introduction to it. The outer contour is set at a density at which individual samples start to merge, as shown by samples outside it. The curves along the right-hand and top sides show the normalized sample-density distribution per axis quantity. The summit of the contour mountain, marked with a cross, represents the most common value pair. Its location may differ from the maxima of the distributions because these pixels do not necessarily combine.

The two dashed curves crossing near the summit specify the first moments per axis bin, for cuts along columns and rows, respectively. They are Cartesian at no correlation (with contours in an elliptical or circular bull's-eye pattern) but come together toward slanted lines at high correlation (to the lower left and upper right) or anticorrelation (to the lower right and the upper left). When the two quantities share the same scales, these 1:1 correlation lines lie along the diagonals.

The labels in the top left corner specify the data set and the time lag between the samplings of the two quantities. The numbers in the top right corner specify the total number of pixel pairs and the density increase factor between successive contours. The numbers at the bottom specify linear Pearson correlation coefficients, at the left for all sample pairs, at the right for the subsets constituting the four quadrants of the diagram around the mountain summit. At high linear correlation or anticorrelation, the latter are close to the overall coefficient along the corresponding

\footnotetext{
http://www.staff. science.uu.nl/ rutte101
} 

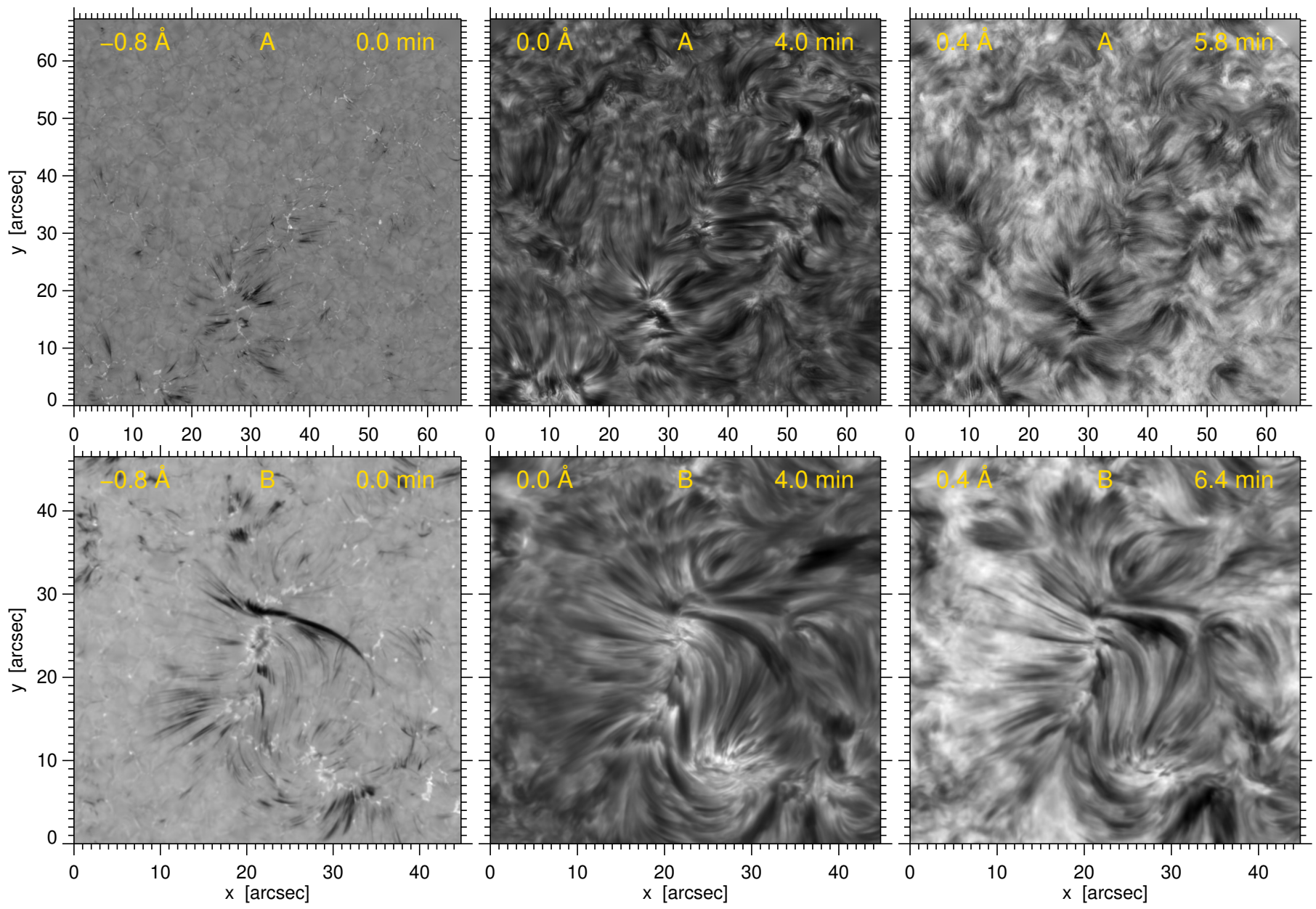

Fig. 1. Full-field overviews for data A (upper row) and data B (lower row). First column: H $\alpha$ blue-wing intensity at $\Delta \lambda=-0.8 \AA$. Second column: $\mathrm{H} \alpha$ line-center intensity 4 min later. Third column: $\mathrm{H} \alpha$ red-core intensity at $\Delta \lambda=+0.4 \AA$ about 6 min after the first column. Each panel is bytescaled independently. The data A images start at the best-seeing moment. The data B images sample the Pub B PHE, contrail fiber, and return aftermath. The timings are marked by triangles in Fig. 2. Online blinkers for these images (for page flipping per pdf viewer in single-page, fit-to-page, or full-screen mode): 1-2, 1-3, 4-5, 4-6).

diagonal direction; they are small in the other direction. These partial quadrant coefficients often furnish better quantification for different agents than the overall coefficient, but likewise they are strongly weighted toward the high densities near the summit. The degree of association at low densities is indicated best by the angle between the outer parts of the moment curves, but rare features may affect outer contours only locally.

In various figures including the last panel of Fig. 5, we apply the format not to single-image comparisons (instantaneous or time-delay pairs) but to the whole data sequences or their best-seeing subsets. Each successive image pair then represents an additional set of pixel comparisons, which increases the statistics. Long-lived or often-occurring pair combinations earn higher density in this temporal summing.

Showex browser. Our main activity in this study consisted of detailed inspection of the $\mathrm{H} \alpha$ data searching for line-center contrail fibrils and return aftermaths after RBEs by blinking enlarged parts of the spectral $\mathrm{H} \alpha$ images while varying the sample wavelengths and time separations. In this case, we did not use the CRISPEX browser of Vissers \& Rouppe van der Voort (2012), but a new one called SHOWEX that was developed by the first author and is available online ${ }^{2}$. It is less versatile

\footnotetext{
2 http://www.staff.science.uu.nl/ rutte101
}

than CRISPEX in not showing spectral time charts and offering fewer measurement tools, but it permits fast blinking with sliders modifying sampling wavelengths and time delays, offers easy zoom-in to detail, permits temporal averaging, and accepts many different files of different types. It also shows instantaneous scatter plots in the Strous format, with live marking of the scatterplot location for the pixel pair under the cursor in one of the images. Figure 3, its online movie versions, and the online image blinkers for many figures represent shorthand emulations of our extensive inspections.

\section{Results}

Our results consist of figures that we first describe one by one while deferring their overall interpretation to Sect. 4.

Figure 1: we start with full-field samplings of data A and B to show that both data sets targeted patches of somewhat active network in quiet-Sun areas. Both were near disk center. The upper half of the data A field was mostly network free and resembles the quiet-Sun area in the upper left part of the field studied by Rouppe van der Voort et al. (2007).

The sample timings in Fig. 1 are specific selections. For data A the first panel is at the moment with best seeing (Fig. 2), with its central part enlarged in the second panel of Fig. 2 to 

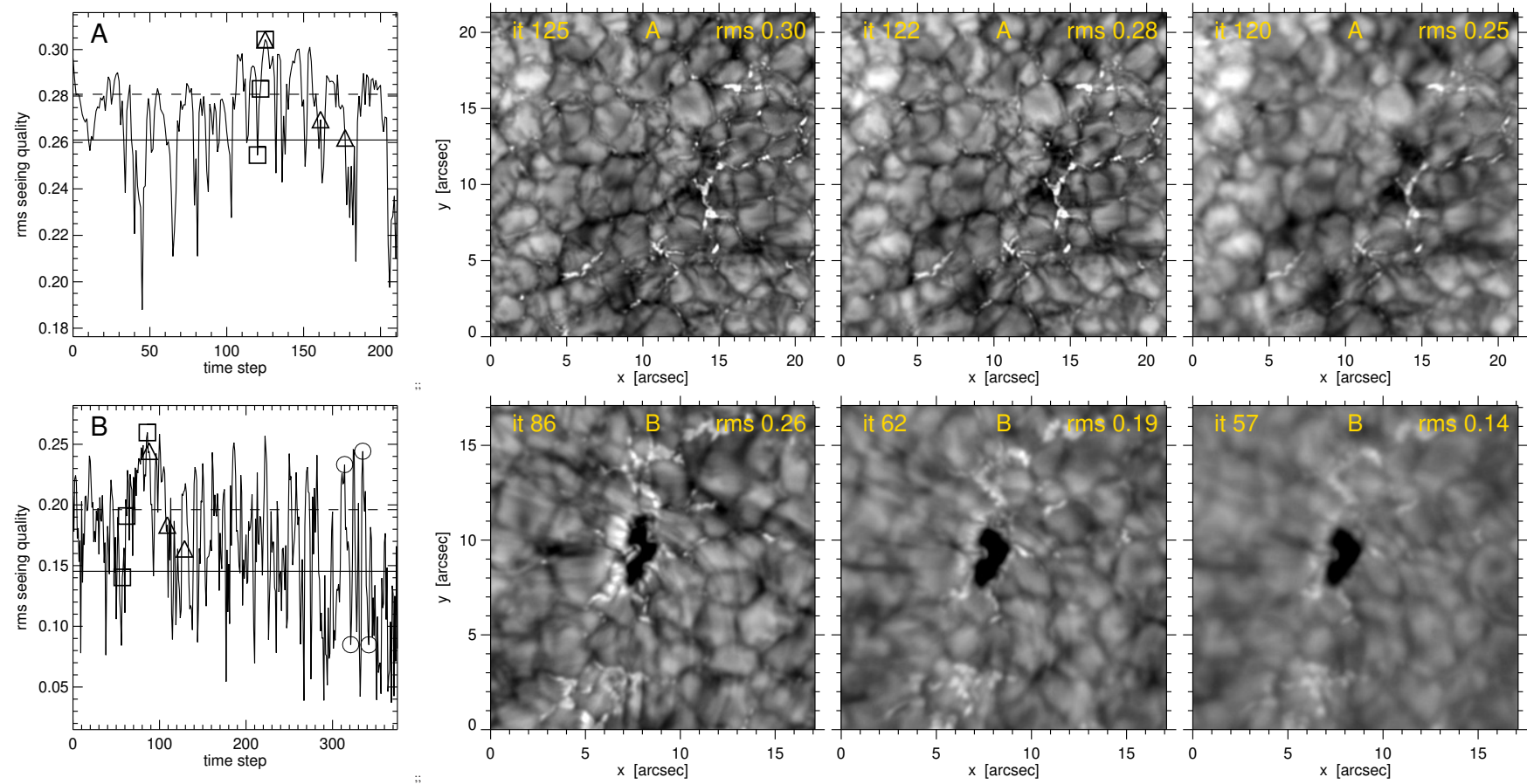

Fig. 2. Seeing quality for data A (upper row) and data B (lower row). First column: root mean square contrast variation against time for the wideband $\mathrm{H} \alpha$ image sequences. The rms units are arbitrary per data set. The solid horizontal lines show thresholds for discarding the worst images ( $25 \%$ for data A, $33 \%$ for data B). The dashed lines show the mean rms for the remaining images. Other panels: corresponding image center cutouts at the best seeing, seeing near the mean-above-threshold value, and seeing near the threshold value, sampled at the times marked by three squares in the graphs that are close for sampling the same solar scene. The three triangles in the upper graph mark the samples in the upper row of Fig. 1. The three triangles in the lower graph mark the samples of the Pub B PHE, contrail fibril, and return aftermath shown in Figs. 1 and 7. The two pairs of circles at the right mark 4 min delay pairs at good and poor seeing used in Fig. 7. The image greyscales along rows share the clipped byte scaling of the sharpest images at the left, including saturation of the pore in the lower row to boost granular contrast.

show its quality. The second and third are for reasonably good seeing moments 4 and $6 \mathrm{~min}$ later, respectively. For data $\mathrm{B}$ the first panel shows the Pub B PHE, the second panel the subsequent contrail fiber $4 \mathrm{~min}$ later, and the third panel the return aftermath 6 min later.

The three lower panels represent a concise summary of Pub B. The visibility of the striking PHE at left at the AIA 304, 171,193 , and $94 \AA$ wavelengths implies full hydrogen ionization because the corresponding formation temperatures are well above the $20000 \mathrm{~K}$ value at which hydrogen ionizes fully in coronal equilibrium (at lower temperature for higher density, see Fig. 6 of Pub B). Minutes later, the $\mathrm{H} \alpha$ core showed a fat dark fibril along its track (second panel). Just below it, an adjacent one appeared as well, made by a preceding shorter PHE called "contrail B" in Pub B. Both retracted in their return aftermath with considerable $\mathrm{H} \alpha$ core redshift (third panel).

Our quest here is to determine whether the less spectacular but more common PHEs that are visible as RBEs produce smaller $\mathrm{H} \alpha$ core fibrils in a similar manner. Both data samples in Fig. 1 show RBE candidates in the first column. Many seem to have corresponding darkenings in the 6 min delay third column, seen best with the blinkers in the online material.

Figure 2 shows the seeing during the two observation periods. As usual, it varied considerably. We must account for these variations because we applied statistical measures to the whole sequences in the form of temporal averaging and scatter correlation, whereas the detection of slender RBEs and aftermath features is very sensitive to image sharpness.

Figure 2 shows our choice to discard the worst quarter of all images for Data $\mathrm{A}$ in whole-sequence analysis and the worst third for Data B, which had slightly lower quality, as evident in the image samples. The rightmost images show that even these no-pass thresholds still correspond to image quality that may be deemed good at most other solar telescopes.

Figure 3 shows examples of Pub A RBEs, denoted $a-g$ in the seven rows. These seven cases were selected from the 608 RBE skeletons detected in Pub A per visual SHOWEX inspection favoring good seeing and RBE isolation for less confusion. Many others show yet more confused and confusing scenes, but the cleaner examples in Fig. 3 are already a good demonstration of the utter richness, dynamic character, and small scales that are typical for $\mathrm{H} \alpha$ fibrils around network. The online movie versions show such cutouts for each case at the three selected wavelengths while covering all time steps during a $[-2,+6]$ min range around the Pub A skeleton determination.

Each skeleton is shown by a white line at the centers of the blue-wing image cutouts in the first column. The skeleton midpoint was used as center for the cutout per case and is marked with a plus, as a spatial reference also in the other panels per row and in the other movie frames.

The blue-wing samplings are closer to line center than the $\Delta \lambda=-1.3 \AA$ used for the skeleton detections in Pub A in order to show the RBEs fatter and longer around these. When the same RBE was detected as a Pub A skeleton in multiple time steps, we selected the RBE with the best seeing at its detection time and the shown delay times.

The second column shows the line-center scene two minutes earlier. Case $a$ shows a dark bunch of fibrils at the left that were likely created just before and vanished with time (other panels). At poor seeing (fourth panel) it seemed a thick homogeneous 
R. J. Rutten et al.: Solar $\mathrm{H} \alpha$ features with hot onsets. IV.
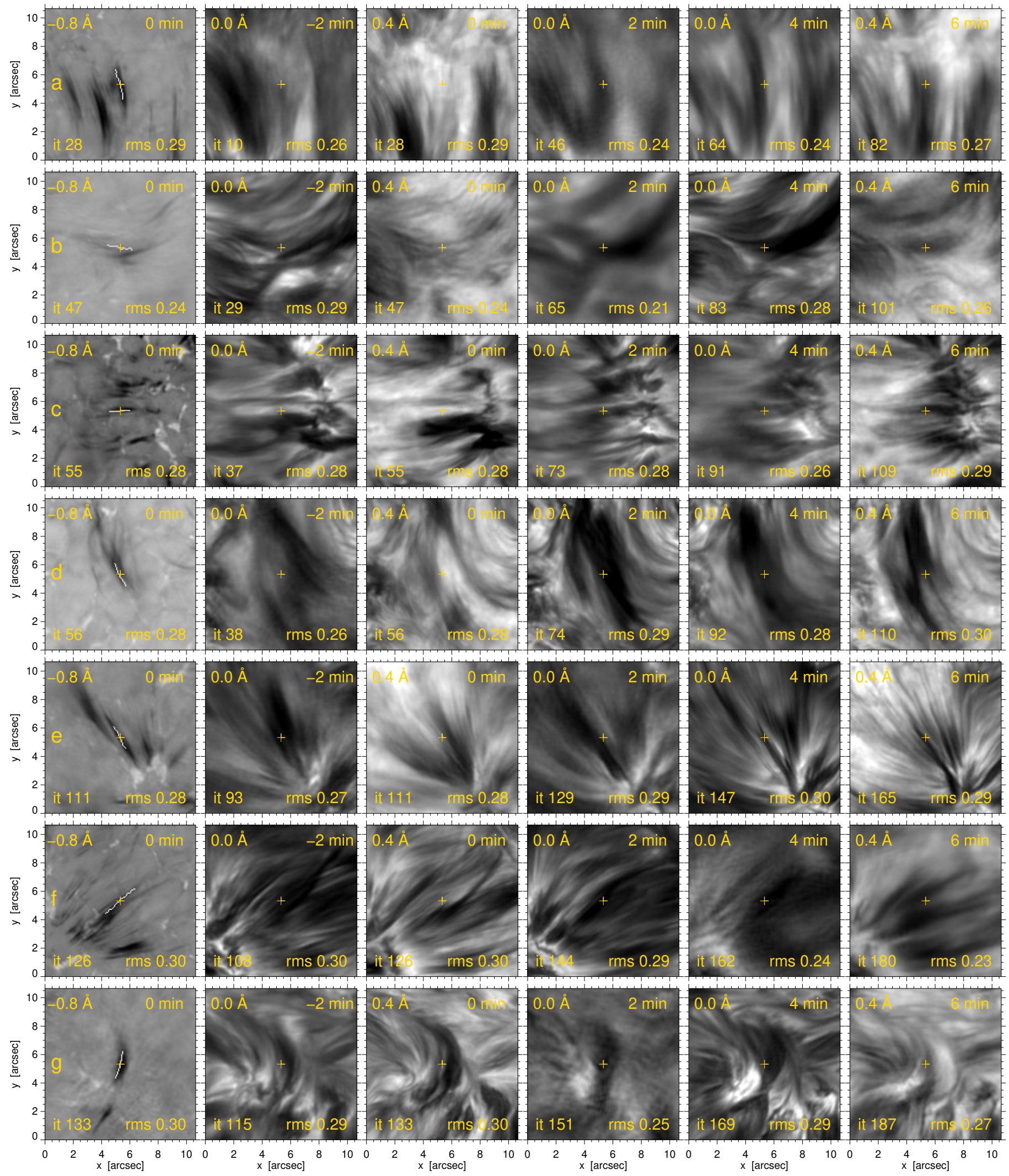

Fig. 3. Example sequences of RBEs and their aftermaths from data A in the form of $\mathrm{H} \alpha$ image cutouts. The images can be zoomed in per pdf viewer and the online movie versions can be inspected and enlarged. The $\Delta \lambda$ and $\Delta t$ values are specified at the top of each panel, the time steps (it) and seeing quality (rms) are at the bottom. Rows: seven cases $a-g$ in time order. First column: RBE skeleton taken from Pub A (white) overlaid on a cutout of the $\mathrm{H} \alpha$ blue-wing image at $\Delta \lambda=-0.8 \AA$ from mean line center. The cutout is centered on the skeleton center marked with a plus. Other columns: $\mathrm{H} \alpha$ cutouts at $\Delta \lambda=0.0$ or $+0.4 \AA$ from mean line center at time delays $\Delta t=-2,0,2,4,6$ min with respect to the RBE measurement. The byte scaling is defined per row and per wavelength by the image with the best rms value. The online material includes three-panel movies per case showing all pertinent time steps at the three sample wavelengths. Direct links per case: $a, b, c, d, e, f, g$. 


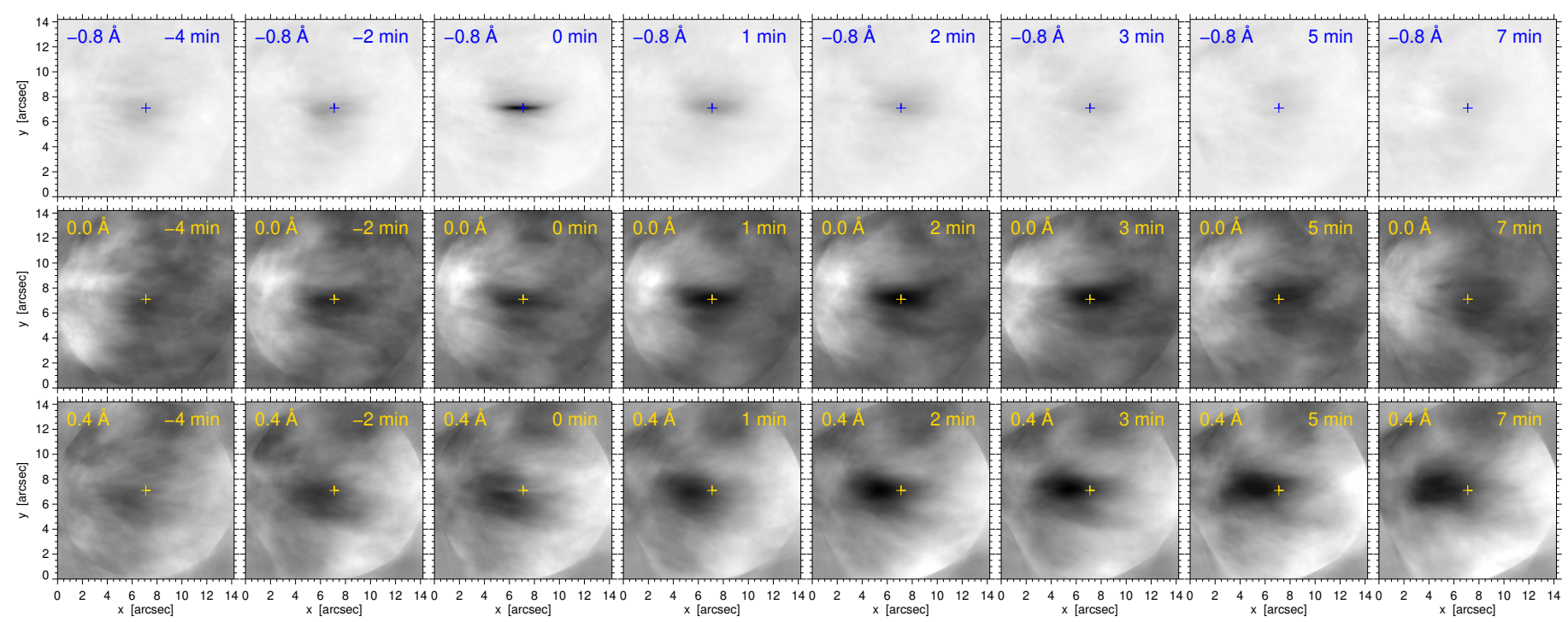

Fig. 4. Stacked $\mathrm{H} \alpha$ image cutouts for data A. Before addition, all seeing-passed image cutouts are centered on the center of the RBE skeleton (plus) and rotated to point the skeleton to the right, away from the network to the left. Rows: averaged $\mathrm{H} \alpha$ samplings at $\Delta \lambda=-0.8,0.0$ and $0.4 \AA$ from mean line center. Columns: averaged samplings at different time delays with respect to each RBE track, from 4 min earlier sampling at the left to $7 \mathrm{~min}$ later sampling at the right. The $\Delta \lambda$ and $\Delta t$ values are specified at the top of each panel. The greyscales are shared along rows, defined by bytescaling the $\Delta t=0,2,3$ min panels, respectively. Blank corners result from cutout rotations.

fibril, but at the best seeing (third panel) it appeared finely striated. Case $e$ has a similar bunch at the skeleton position and timing.

The third column is simultaneous with the RBE in the first column and also samples the $\mathrm{H} \alpha$ core, but not at line center but at $\Delta \lambda=+0.4 \AA$ to emphasize redward line shifts. It also displays the blueshifts that are part of the RBE phenomenon in the form of cospatial brightenings. These show up as thin bright stripes, thinner than the blue-wing RBEs (the reason is discussed in Sect. 5). They duplicate the skeletons in the first column in their location (through the crosses) and orientation, but they extend farther. Such thin bright RBE stripes are seen in all seven panels of the third column, but they are near the SST resolution limit and only visible at the best seeing so that they are not a robust alternative to blue-wing RBE detection. In these best-seeing samples they suit better than the skeletons for comparison with the delay images to the right. These are at $\Delta t=2,4,6 \mathrm{~min}$, the first two for line center to check for contrail fibrils, the last one at $\Delta \lambda=+0.4 \AA$ to check for return aftermaths with core redshifts as in Fig. 4 of Pub B.

We discuss the seven cases one by one. In case $a$ (movie), the 0 min panel (third in the top row) has no dark fibril at its center, but two bunches to the sides that may be related to previous RBE activity, as suggested by other RBE locations and directions in the first panel. The three delay panels show a dark fibril, likely a striated bunch, but the $2 \mathrm{~min}$ and 4 min seeing was less good. It has shortened in the 6 min panel, suggesting contraction, as in the Pub B return aftermath.

In case $b$ (movie), the thin bright stripe in the third panel suggests that the skeleton at the left is part of the long curved thin gray feature. This obtains a dark fibril around it at 4 min delay, with a weaker and shorter remnant at 6 min delay.

In case $c$ (movie), the thin bright stripe in the third panel is part of a larger blueshift feature pointing left that was also present two minutes before. It neighbors a dark fibril bunch below it that is already gone in the 2 min panel, possibly replaced by new (RBE?) blueshift. The best indication of a fibril-after-RBE is in the $6 \mathrm{~min}$ red-core panel, retracted to the right of the plus.
In case $d$ (movie), the thin bright stripe in the third panel curves to the bottom right, followed by a wider fibril bunch and replicated as a thin dark stripe in the rightmost panel. The other nearby features illustrate how easily these scenes become confused.

Case $e$ (movie) already shows a fibril around the plus in the second panel, which is mostly gone in the next panel, then a dark bunch at $2 \mathrm{~min}$ that retracts at $4 \mathrm{~min}$, and a long, thin aftermath feature in the last panel. There is suggestive correspondence between dark features in the 4 min panel and the RBE panel at left.

Case $f$ (movie) is again one in which it seems best to compare the thin bright curved stripe in the third panel with the fibrilar darkening in the later panels. The same holds for case $g$ (movie), in which the curved bright stripe seems to become replicated as a dark strand in the 4 min panel; it retracts in the last panel.

None of these cases is as obvious and straightforward as the PHE, contrail fibril, and return aftermath of Pub B. However, they all suggest roughly similar behavior in their development. When the dark features around the pluses in the first and the other columns are compared, they seem to match better at $4 \mathrm{~min}$ delay than between the simultaneous first and third columns. Yet better matches occur between the thin, bright RBE stripes in the third column and slender dark features in the last column. Lack of one-to-one dark-dark similarity between RBEs and line core fibrils was already remarked in Pub A (caption to its Fig. 3). The examples in Fig. 3 suggest that better similarity is reached at multiple-minute delay in core imaging, but with much competition from neighboring similar features in other development stages.

This is yet more evident in the online movie versions of these seven cutout areas. They vividly illustrate that at high angular and temporal resolution the $\mathrm{H} \alpha$ chromosphere around network consists of a bewildering multitude of fast-changing small features for which direct cause-and-effect identification is much impeded by similar competitors and also by intervals of worse seeing. 

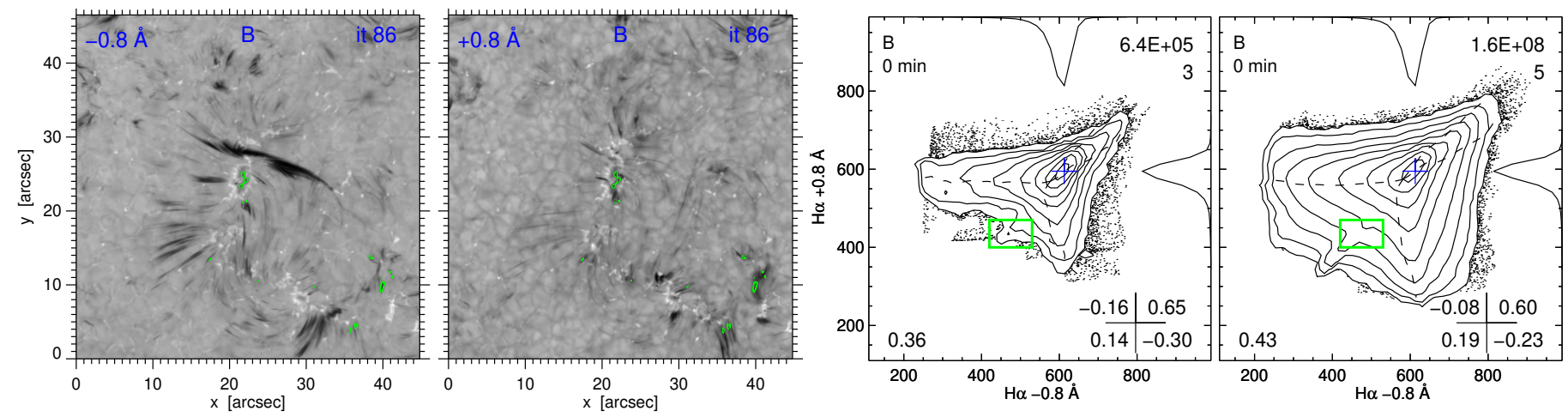

Fig. 5. Strous-format scatter analysis for data B. Images: simultaneous blue- and red-wing images at $\Delta \lambda= \pm 0.8 \AA$ at the best-seeing moment (highest square in the lower graph of Fig. 2). First diagram: scatter contours for this image pair. The format is detailed in Sect. 2. The axis quantities are intensities in SST data units. The green box selects a specific low-density contour feature with the corresponding pixels outlined by green contours in the images. Second diagram: scatter contours for all such 252 image pairs in the data B sequence passing the seeing threshold, with the same axes and the green box repeated as location reference. Online image blinker: 1-2.

Figure 4: in view of the small-scale confusion evident in our browsing with SHOWEX and illustrated in Fig. 3 and its movie versions, we now turn to statistical full-duration analyses of the data sequences, excluding their worst seeing samples ( $25 \%$ and $33 \%$, respectively). Figure 4 is made from the data A $\mathrm{H} \alpha$ sequence and the Pub A skeleton list by cutting out subimages centered on the midpoint of each skeleton and rotating each subimage so that all skeletons point to the right, emanating from network to the left. All subimages passing the seeing threshold of Fig. 2 are then summed to obtain averages. The number of summed pairs varies between 200 and 300 depending on the seeing sampling and on delay values exceeding the observing duration.

As expected, the top row shows RBEs averaging to a dark slender horizontal feature at 0 min delay. It is also weakly visible in the adjacent panels. This presence might be attributed to RBE longevity, but the RBE lifetime histograms in Fig. 13 of Pub A, Fig. 4 of Sekse et al. (2012), and especially in Fig. 4 of Sekse et al. (2013b) show that RBEs typically last only half a minute, rarely above a minute. Our SHOWEX inspections and Fig. 14 below suggest that this weak presence is instead due to $\mathrm{RBE}$ recurrence, in agreement with Sekse et al. (2013b), who found that on average, RBEs occur every $84 \mathrm{~s}$ at locations around network where at least one appeared during their observation. Such fast RBE repetition contributes confusion in Fig. 3 and apparent longevity here.

The second row shows an elongated RBE signature in the 0 min panel because RBEs tend to have core darkening (first column of Fig. 13). The -2 min panel shows a similar but vaguer feature, similar to the top row, which we also attribute to frequent RBE recurrence. Subsequently, darker elongated correspondence clouds with the same horizontal orientation develop during 1-3 min delay and then become more diffuse. These imply that statistically, there is significant probability for fibril-shaped RBE-aligned core darkening after RBEs. If this were not the case, these averaged image cutouts would instead be smoothly gray or show vague blobs, as in the -4 and +7 min panels.

In the bottom row the thin, bright RBE stripes in the red-core images in the third column of Fig. 3 add up to produce a bright thin horizontal feature in the third panel, longer and thinner than the RBE feature in the third panel of the top row, but representing the same phenomenon. In the later samplings the elongated black blob darkens as in the second row. It extends left of the plus due to fibril retraction back to the network root in return aftermaths.
There are vaguer and more roundish blobs in the first and last columns of all three rows, with the one in the last panel still quite dark and similar ones in the -2 and 0 min panels of the bottom row spreading around the thin bright RBE stripe in the 0 min panel. We attribute these to close-lying companion RBEs at different times. Our SHOWEX inspections, the examples in Fig. 3, and the studies of Sekse et al. (2012, 2013b,a) show that RBEs are often launched close together with similar orientation, for example the pair in Fig. 10 of Sekse et al. (2013b). Frequent adjacency adds to frequent recurrence as darkener in these image summations.

Figure 5: we now turn to Strous-format scatter correlations. This figure introduces the format with a relatively straightforward comparison from data B in which well-known agents define the solar scenes. The images sample the outer $\mathrm{H} \alpha$ wings at equal wavelength separation from line center $(\Delta \lambda= \pm 0.8 \AA)$, simultaneously at the best-seeing moment. Their blinker in the online material helps to appreciate their correspondences and differences.

The first diagram shows the corresponding Strous-format scatter correlation. The second diagram results from treating all instantaneous image pairs in the data B sequence above the seeing-quality threshold of Fig. 2 likewise. The increased statistics then give smoother contours that sample steeper slopes and spread farther from the summit.

The main agents sampled in the outer $\mathrm{H} \alpha$ wings are the granulation in the deep photosphere, which covers most of these fields and dominates the summit contours, magnetic bright points, which result in the narrow bright-bright mountain ridge pointing to the upper right, and RBEs in the blue wing and RREs in the red wing, which cause the wider ridges from the summit leftward and downward. Lesser agents such as the $p$-mode Dopplershift pattern cause further blurring. There is no signature of reversed granulation, gravity waves, internetwork shocks, magnetic canopies or other upper-photosphere or chromospheric structures because $\mathrm{H} \alpha$ has an extinction gap between the deep photosphere and the chromosphere (Schoolman 1972; Rutten \& Uitenbroek 2012) and senses the latter only closer to line center in other fibrils than RBEs and RREs (including the long ones studied here).

The appearance of the granulation in the two images differs through the intensity-Dopplershift correlation (bright granules rise, dark lanes subside), which doubly shifts each wing accordingly through the fixed passband. This asymmetry diminishes 
granular contrast in the blue wing, enhances it in the red wing, and defines the shape of the mountain summit with near-parallel moment curves. It also enhances bright-point contrast in the blue wing (Leenaarts et al. 2006); additional blue-wing bright-point enhancements result from commonly occurring downdrafts in magnetic concentrations (Leenaarts et al. 2010). They tilt the bright-point scatter ridge clockwise from the diagonal direction. It widens slightly in the full-sequence diagram, with a lower upper-right quadrant Pearson coefficient, because all additional images are less sharp.

The Pub B PHE in the first image contributes heavily to the first diagram with very dark blue-wing pixels, but no signature in the red wing. However, its contribution to the second diagram is negligible because it lasted only briefly; when its time segment is removed from the summation, the contours and coefficients remain virtually identical. In this diagram the leftward ridge is wider than the downward ridge because there are more RBEs than RREs (Sekse et al. 2013a). RBEs also reach darker extremes. These features are mutually exclusive because the required core Dopplershift is blueward for RBEs, redward for RREs, and makes these chromospheric features transparent in the other wing so that it samples the granulation far underneath. The darkest pixels in one sample the distribution for the other without preference; the moment curves return to the distributionmaximum locations.

The green box in the first diagram emulates SHOWEX identification of which pixels cause what scatter-diagram feature. The corresponding green-contoured pixel pairs in the images show that most of the unusual-looking extension of the outer contour came from the single pore near image center. It covered only a promille of the field so that it shows up only beyond the sixth contour from the summit $\left(3^{-6}=0.0013\right)$. Through its longevity during the sequence, it gained a much clearer signature in the time-summed diagram at the right: a conspicuous narrow high-correspondence ridge with $45^{\circ}$ response equality. Masking the pore produced the same diagram without this ridge. Its presence would remain the same if the pore had moved around or had appeared at a random location per image.

This example of Strous-format scatter diagram production demonstrates that the full-sequence variant is useful to show and disentangle statistical properties for multiple agents that occur briefly but frequently (such as granulation, RBEs, and RREs) or more persistently (such as magnetic bright points and the pore), even at small or minute filling factor (such as the pore).

In this multi-agent comparison the linear overall Pearson correlation coefficient is a useless estimator. The quadrant coefficients do better but remain too biased toward the summit. This is evident for the lower left one, which has relatively high values from the initial correspondence near the summit where the moment curves cross before becoming nearly no-correlation Cartesian. Masking the pore reduces the full-sequence lower left value only from 0.19 to 0.18 and does not affect the moment curves. The other two quadrant coefficients are also set by the tilt of the summit contours. Inspection of the shape of the lowdensity outer contours is much better for recognizing correlation signatures of different nondominating but persistent or recurring agents, as demonstrated by the pore.

Finally, this figure suggests that RBEs and RREs might be used in tandem to increase spicule II statistics, for example by selecting the darkest pixels in either wing per time step. However, in many figures we compare RBE presence to later core redshift in searching aftermaths with return flows. Because RREs also have core redshifts, ambiguities would then arise between them and their aftermaths. We therefore restrict most figures to RBEs only.
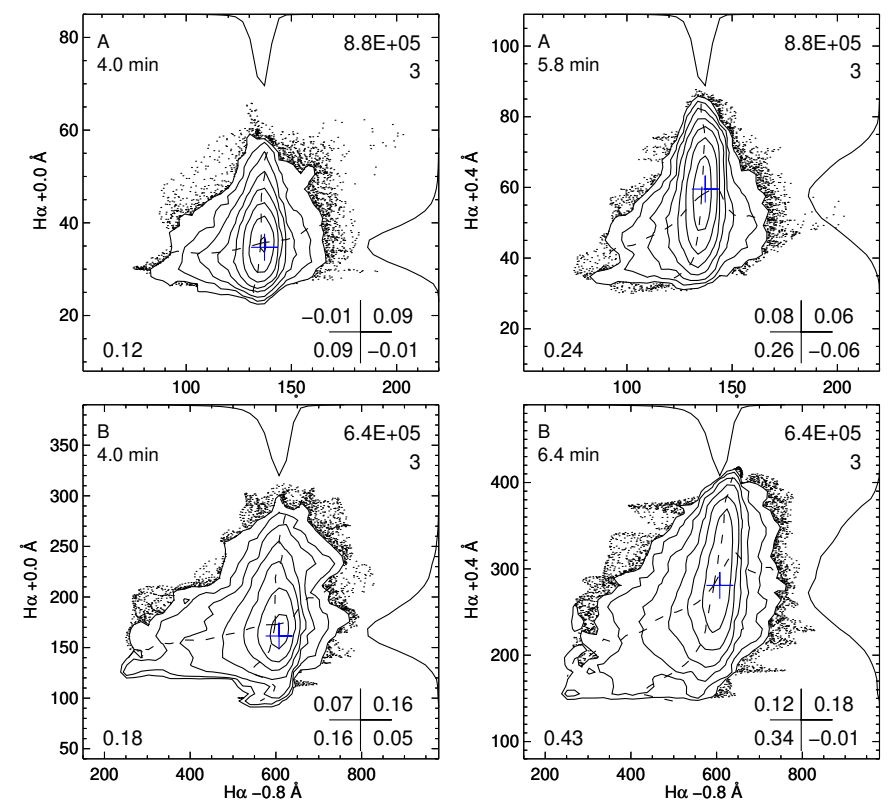

Fig. 6. Scatter diagrams for the data A (upper row) and data B (lower row) image pairs in Fig. 1. Left column: intensity at the line center of $\mathrm{H} \alpha$ at $4 \mathrm{~min}$ time lag against intensity in the blue wing at $\Delta \lambda=-0.8 \AA$ 4 min. Right column: intensity at $\Delta \lambda=+0.4 \AA$ at about 6 min lag against intensity in the blue wing.

Figure 6 initiates our time-delay scatter analysis by employing it to the six images in Fig. 1. We there remarked that their inspection suggests correspondence between RBE presence in the first column and later red-core darkening in the third column. Here this apparent correspondence manifests itself as extensions ("spurs") of the contour mountain left and down from the summit.

The first contours from the summit suggest no correspondence except for the slight tilts in the last diagram. Figure 1 shows that this lack of correspondence is dominated by the very quiet internetwork sampled especially in the upper part of data A. It has no RBEs but only granulation in the $\mathrm{H} \alpha$ blue wing, and it is poor in fibrils in the $\mathrm{H} \alpha$ core.

The outer contours instead show left-down mountain spurs. In data B the Pub B PHE, contrail, and aftermath contribute to these, making them more pronounced with higher lower-left Pearson quadrant coefficients. The spurs are most pronounced in the red-core diagrams at the right and show large dark-dark correspondence. For example, in the final diagram, all RBE pixels darker than 400 data units were also darker than average 6.4 min later in the red-core image, with the darkest followed by darkest. However, there were also about as many darkest red-core pixels with slight or no preceding wing darkening, and in the left-hand diagrams there are yet darker line-center pixels without preceding wing darkening. Below we identify RREs and dynamic fibrils as contributors to these.

Figure 7 shows the effect of degraded resolution on scatter diagrams. The images in the first column are the same as the first data B images in Fig. 1, sampling the Pub B PHE and contrail fibril, with the scatter diagram underneath repeating the lower left diagram in Fig. 6 for reference.

The green box selects the spur part of the contour mountain in the first diagram, with the corresponding pixels outlined by green contours in the images above. All belong either to the Pub B PHE or to RBEs pointing away from the network. This 

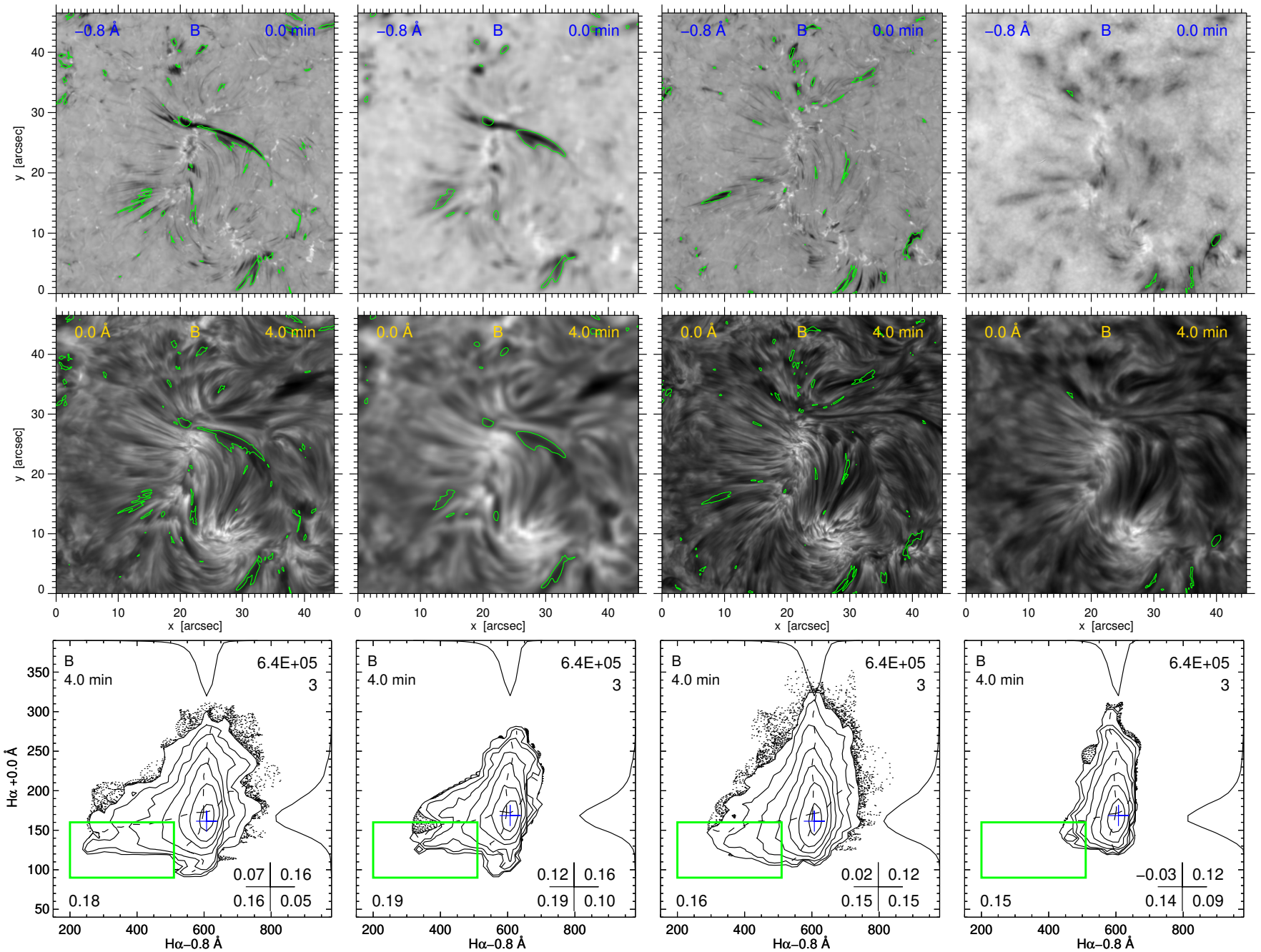

Fig. 7. Time-delay scatter analysis at good and poor resolution in data B. First row: images in the blue wing of $\mathrm{H} \alpha$ at $\Delta \lambda=-0.8 \AA$. Second row: images at $\mathrm{H} \alpha$ line-center taken 4 min later. Third row: scatter diagrams for each image pair above with the same axes as in Figs. 6 and 8 . The green box selects the lower left part with corresponding pixels outlined by green contours in the images above. First column: image pair for the Pub B PHE and contrail, the same as in Fig. 1. Second column: same image pair smeared over 1 arcsec. Third column: image pair with good seeing. Fourth column: image pair with poor seeing. The latter pairs are marked with circles to the right in the lower graph of Fig. 2.

part of the first scatter diagram therefore represents RBE signatures including the Pub B PHE.

The second column treats the same images, but with 1 arcsec smearing. The contour spur shrinks considerably toward the mountain summit. The image contours show correspondingly that far fewer RBEs now qualify as part of the scatter spur.

The third and fourth columns are similar demonstrations for the pair of good-seeing images and the pair of poor-seeing images marked by circles to the right in the lower graph of Fig. 2. At each wavelength, the samplings were within $1.5 \mathrm{~min}$ of each other so that the scenes differ primarily in resolution. In the good-quality third-column panels the green box selection again selects RBEs (now without the extraordinary Pub B features), as shown by the contours in the images above, which mostly display elongated shapes. In the final poor-seeing pair the RBE selection again diminishes drastically and the scatter spur shrinks much toward the contour mountain summit.

Figure 8 shows scatter correlations combining image pairs from the full data sequences, excluding the worst-seeing samplings. The many samples produce much better statistics than in Fig. 6, with well-defined contours reaching out farther from the mountain summits, as in Fig. 5.
The first and third rows for Data A and B, respectively, chart $\mathrm{H} \alpha$ line-center $(\Delta \lambda=0.0 \AA)$ intensity against blue-wing intensity at $\Delta \lambda=-0.8 \AA$ at different time delays. The second and fourth rows chart $\mathrm{H} \alpha$ red-core intensity at $\Delta \lambda=+0.4 \AA$ against blue-wing intensity.

The data B $\Delta t=+4$ min diagram in the third row (central panel) is the full-sequence version of the corresponding scatter diagrams in Figs. 6 and 7. It shows the same mountain spur to the lower left, implying significant dark-dark association. The box selections in Fig. 7 suggest that most or all of these belong to RBE-like features. The spur starts already at the mountain summit, implying that very many pixels have slightly dark - slightly dark association.

The first two and last two panels of the third row do not show the spur and show nearly perpendicular moment curves, except for the small bright-bright bulge in the horizontal one, which is constantly present and probably represents the network itself (Fig. 1). The main change with delay duration in the third-row diagrams is the downward tilt of the leftward mountain spur during delays $\Delta t=2-6 \mathrm{~min}$.

The contour patterns and development in the first row for data $\mathrm{A}$ are similar to those for data B in the third row. The 

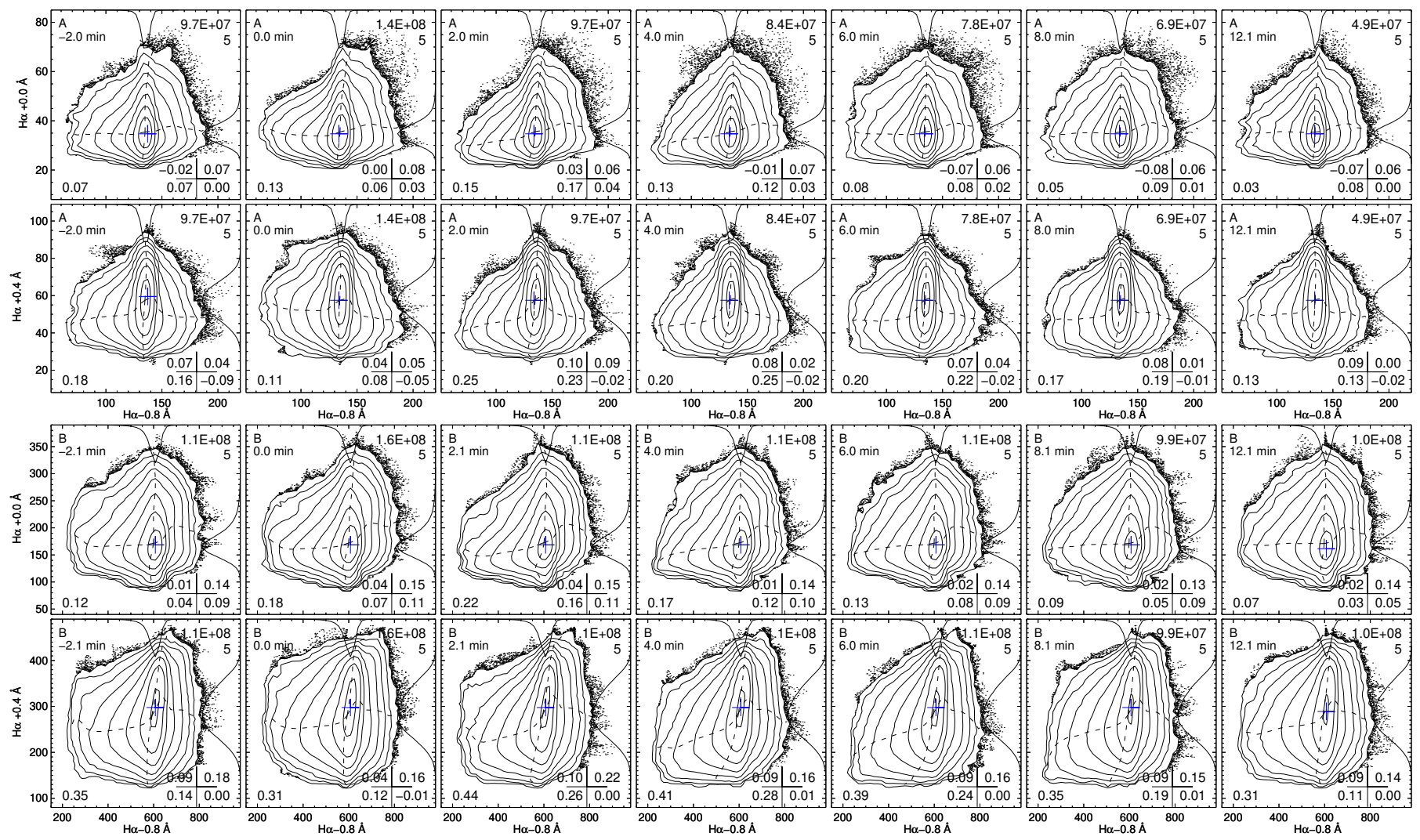

Fig. 8. Full-sequence time-delay scatter analysis for data A (upper two rows) and data B (lower two rows). In each pair the upper row shows the pixel-by-pixel correlation for $\mathrm{H} \alpha$ line-center intensity before or after the $\mathrm{H} \alpha$ blue wing intensity at $\Delta \lambda=-0.8 \AA$. The lower row per pair shows the correlation of red-core intensity at $\Delta \lambda=+0.4 \AA$ with blue-wing intensity. The sampling delays (positive for the core after the blue wing) are specified at the upper left in each panel. The worst-seeing images are discarded (25\% for data A, 33\% for data B). Axes are the same as Figs. 6 and 7.

delayed downward tilt of the leftward spur corresponds to the darkening blob in the central row of Fig. 4.

The second row with sampling at $\Delta \lambda=0.4 \AA$ corresponds to the bottom row of Fig. 4. Compared to the top row, the onion-like contour patterns show an upward shift of the mountain summits due to the very quiet areas in data A, as noted for Fig. 6. The leftand downward spur is clearer in the $\Delta t=2-4$ min panels of the upper row, but in the second row, it persists to longer delay, in agreement with the longer persistence of the corresponding dark blob in Fig. 4.

The fourth row shows the $\Delta \lambda=0.4 \AA$ delay samples of data $B$, which are richer in blue-wing RBEs and core fibrils (Fig. 1). At 6 min delay, the lower left bulge even shows a promontory that persists at $8 \mathrm{~min}$ delay. A bulge signature remains even at 12 min delay.

The two data $\mathrm{B}$ rows start with a squarish contour rise at the upper left, implying bright at $\Delta \lambda=+0.4 \AA$ for dark RBE locations. Based on SHOWEX inspections, we attribute this to the thin bright RBE stripes seen in the simultaneous data A samplings in Figs. 3 and 4.

Figure 9: because Fig. 8 exhibits pixel-by-pixel correlations without information on feature membership, in particular whether the dark-dark associations indeed represent fibrils, this figure recovers these signatures by assembling image constructs. The first two columns show data A, the third and fourth show data $\mathrm{B}$. The upper row shows pairs of identical images in which each pixel is the darkest value at $\Delta \lambda=-0.8 \AA$ for the entire sequence duration. There is no need to discard poor seeing because its contrast blurring already disqualifies these images. The second row alternatively shows the intensity at line center at the same time and the darkest value at $\Delta \lambda=+0.4 \AA$ during the $2-5$ min delay after the above pixel sampling. Selecting the darkest during this delay range also favors samples with better seeing. The bottom row shows corresponding scatter diagrams; three have green selection boxes of specific contour features.

Random patterning might be expected for this darkest-pixel selection, the more so for data B with its three times longer duration and increased activity, but the sharp fibrilar detail in both the first (data A) and third image (data B) shows that these very darkest pixels are also arranged in fibrilar alignments. This suggests that adjacent darkest pixels either sample the same RBE or successive ones that follow similar tracks at different times. Below we show that both types contribute, and also that subsequent RBE samples tend to be close in time (Fig. 14). The first image furnishes RBE mappings that extend well beyond the singlesnapshot skeletons shown in Fig. 3. In the third image the Pub B PHE is prominently present over its full area covered in time. Thus, the blue-wing constructs represent renderings of the darkest parts of RBEs during the observing periods.

The delayed red-core images in the second row show high correspondence with the top row images, much more than the simultaneous line-center images. This can be seen in the online panel blinkers and is quantified by the correlation diagrams in the bottom row. The second and fourth are similar to the corresponding rows in Fig. 8, but show tighter dark-dark association in the left-and-down pointing mountain spurs, with much higher lower-left quadrant Pearson coefficients. In contrast, at 

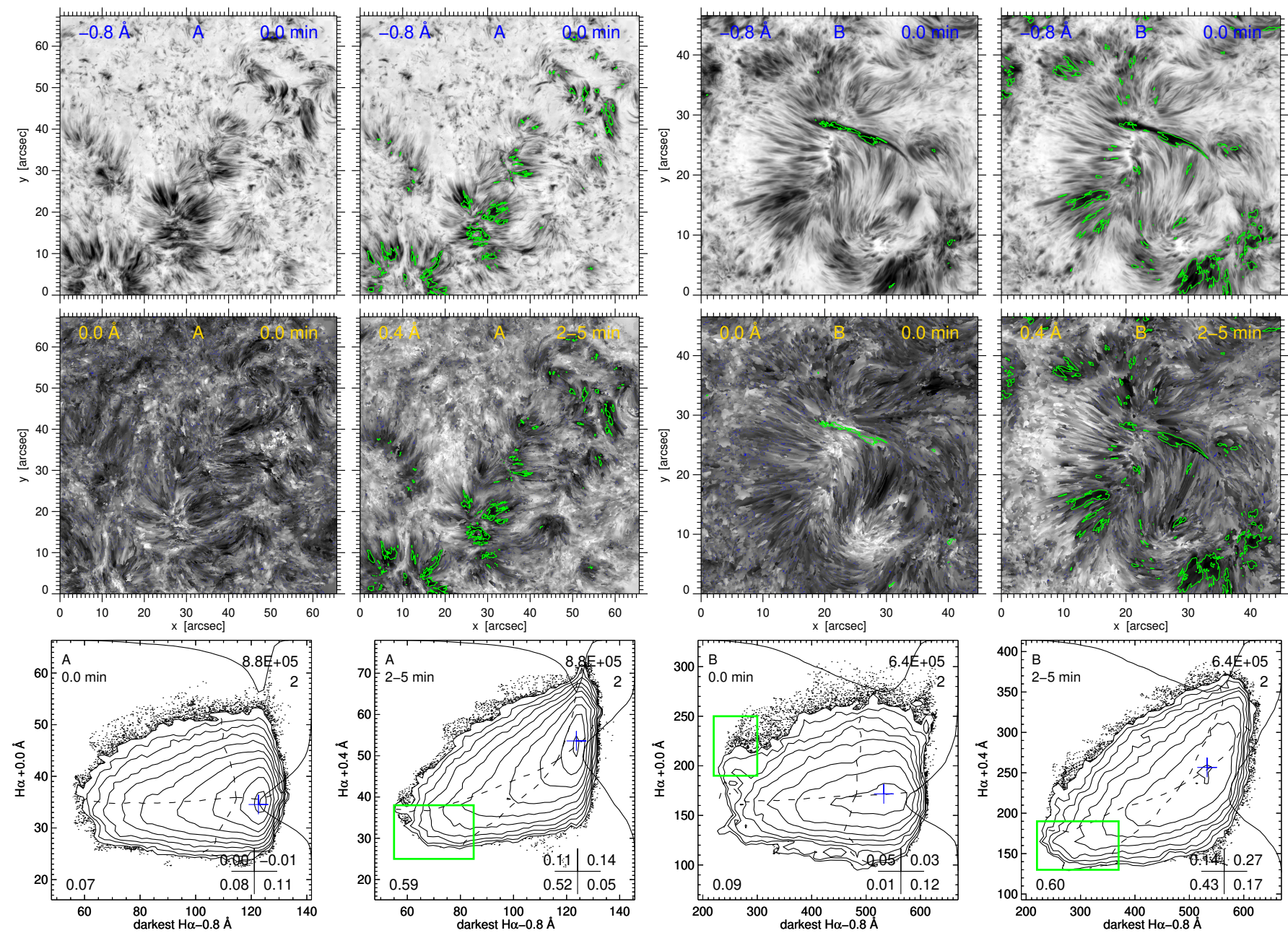

Fig. 9. Time-delay scatter analysis for the darkest RBE moments in data A (left two columns) and data B (right two columns). First row: H $\alpha$ images at $\Delta \lambda=-0.8 \AA$ constructed by selecting the darkest value for each pixel throughout the entire observation duration. The first pair of images is identical except for the green overlays; so is the second pair. Second row: simultaneous and delayed H $\alpha$ line-core images. Third row: scatter diagrams for each image pair above it. First and third columns: data A and B image pairs with the lower image made up of the same pixels at $\mathrm{H} \alpha$ line center at the same sampling times. Second and fourth columns: data A and B image pairs with the lower image made up of the same pixels, but selecting the darkest value per pixel at $\Delta \lambda=+0.4 \AA$ during the 2-5 min delay range after the upper-image pixel sampling times. The green boxes select specific correlation features with corresponding contours in the image pairs. Online image blinkers: 1-5, 1-6, 3-7, 3-8.

simultaneous sampling (first and third diagrams), there is no correlation between RBEs and dark line-center fibrils; the difference between the scatter mountains per pair is striking.

The green-box selection of the darkest-darkest pixel pairs in the second and fourth diagrams shows that the corresponding features are indeed fibrilar in nature and are preferentially located at RBE feet (top images). The green box in the third diagram selects an upward contour promontory in which darkestwing pixels are associated with brighter-than-average line-center pixels. These correspond to brightening by core blueshift in the launch phase of the Pub B PHE.

Figure 10: the left-and-down mountain spurs in the timedelay scatter diagrams in Figs. 7-9 extend from close to the summits of the contour mountains, suggesting that there are very many pixels with slightly dark - slightly dark association. Figure 10 represents a test of their nature, in particular whether they are contributed by quiet internetwork areas without RBEs and fibrils.

The field of data $A$ is best suited to this test because it has wide areas of very quiet fibril-free chromosphere toward its upper left and lower right (Fig. 1). We constructed internetwork and network masks by setting brightness thresholds on a heavily smeared (200 pixel boxcar) average over the whole data $\mathrm{A}$ sequence at $\Delta \lambda=+0.4 \AA$ because the third panel of Fig. 1 shows that this is a suitable divider.

The scatter diagrams in the bottom row of Fig. 10 show minor dark-dark association for the internetwork area, whereas the network diagram is closely similar to the second diagram in the bottom row in Fig. 9, but with less upward spread above the summit that was contributed by the internetwork, as seen in the first diagram. Hence, most pixels contributing slightly dark slightly dark association in the spur belong to the network areas, just as the pixels with darkest-darkest association. The latter are also darker in both diagnostics than any internetwork pixel.

Figure 11: Figures 9 and 10 tested whether darkest core pixels follow on darkest blue-wing pixels. We now reverse this question into asking instead to what extent any darkest core pixels relate to preceding RBEs. We also add red-wing RREs to this question.

The identical images in the top row of Fig. 11 represent the darkest moment per pixel at $\Delta \lambda=+0.4 \AA$ during the whole 

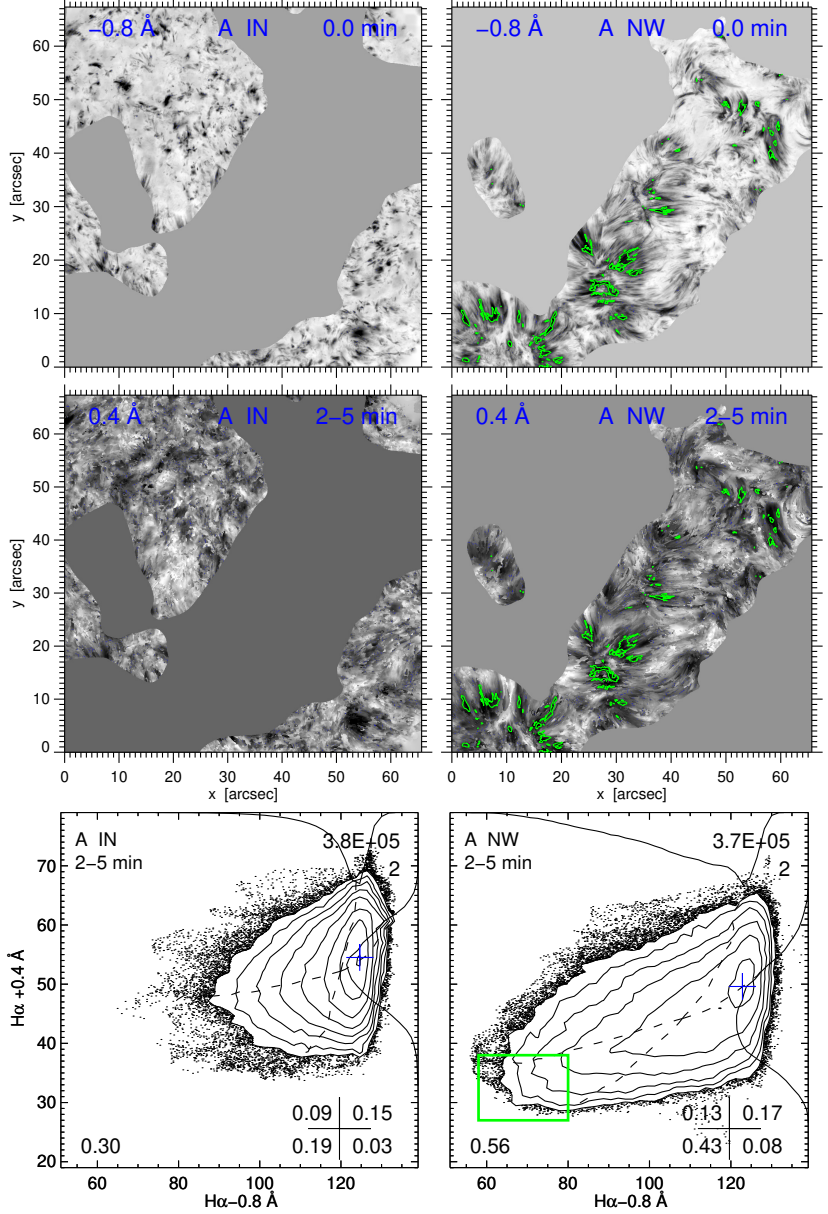

Fig. 10. Time-delay scatter analysis for the darkest RBE moments in data A only, in the same way as in the first columns of Fig. 9, but selecting internetwork (left, label IN) and network (right, label NW). Upper images: darkest value per pixel at $\Delta \lambda=-0.8 \AA$. Lower images: darkest value per pixel at $\Delta \lambda=+0.4 \AA$ during $2-5$ min delay after the upperimage pixel sampling. Scatter diagrams: corresponding pixel-by-pixel correlations. The green box and contours select darkest-RBE and darkest delayed red-core pixels in the network areas. Online image blinkers: $1-3,2-4$.

sequence for network areas only and for data B because this has more network and showed more RBEs and RREs than data A. The network-only mask was constructed similarly to Fig. 10.

The first image of the second row displays the darkest bluewing value per pixel at $\Delta \lambda=-0.8 \AA$ during the 5-2 min time range prior to the above pixel sampling. This image charts preceding RBEs. The right-hand image similarly displays the darkest red-wing value at $\Delta \lambda=+0.8 \AA$ during 5-2 min before to chart preceding RREs. Both images display a multitude of fibrilar features, more and denser in the left-hand image. For each there are correspondences with red-core fibrils in the upper image that are best seen in the column panel blinkers provided in the online material.

The scatter diagrams in the bottom row are now triangular, implying that the darkest red-core pixels have about as much probability to follow on dark RBE pixels at the left or RRE pixels at the right as to follow on non-dark pixels at these outer-wing wavelengths. Some of the latter no-correlation pixels are selected with green boxes and marked by green contours in the images.

RBE and RRE pixels are mutually exclusive, so that when type II spicules cause subsequent core darkening, they contribute
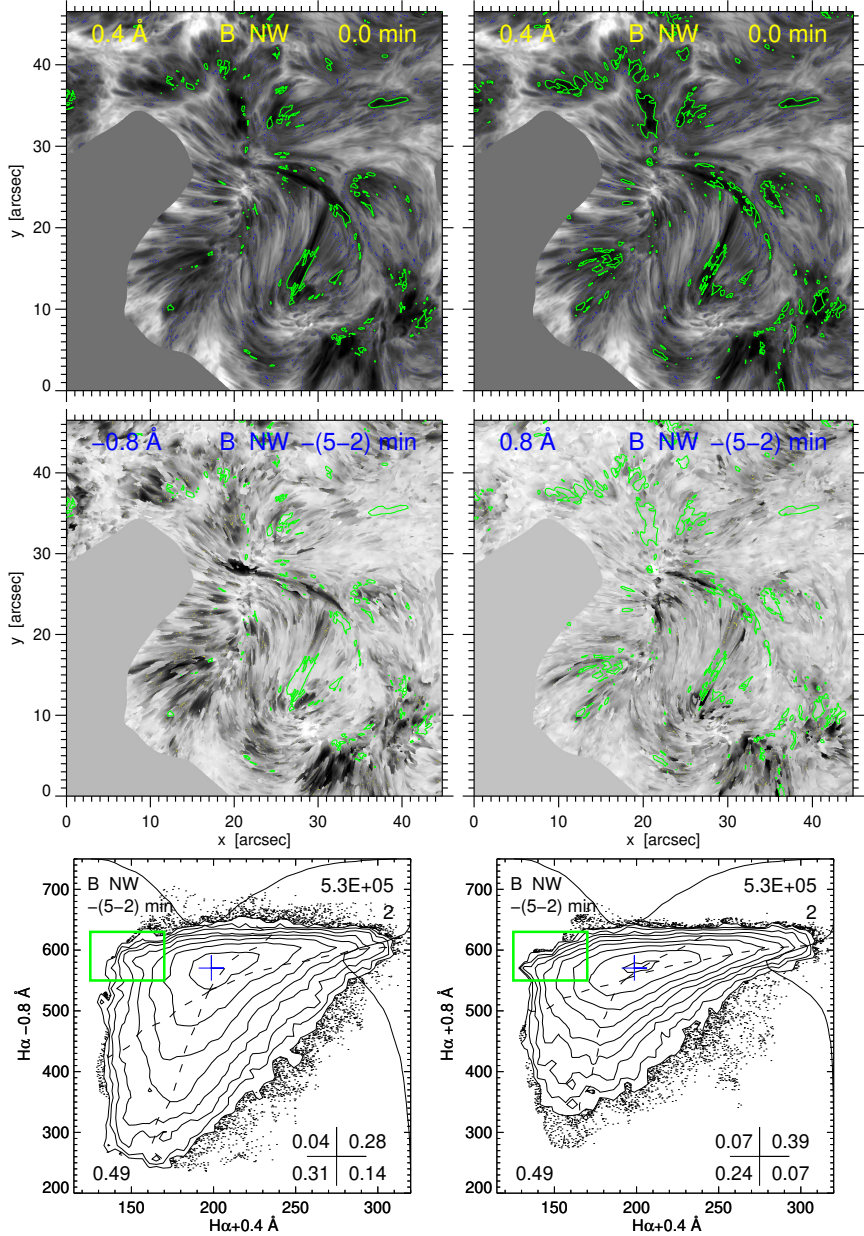

Fig. 11. Time-delay scatter analysis in reverse of Fig. 10 for the data $B$ network only. Upper images: darkest-pixel core samplings at $\Delta \lambda=$ $+0.4 \AA$. Only the green contours differ. Lower images: at the left we show the darkest value per pixel at $\Delta \lambda=-0.8 \AA$ to select preceding RBEs during 5-2 min before the upper-image pixel sampling times, and at the right we show this at $\Delta \lambda=+0.8 \AA$ to select preceding RREs. Scatter diagrams: corresponding pixel-by-pixel correlations. The green boxes and contours select darkest red-core pixels without preceding blue-wing or red-wing darkening, respectively. Online image blinkers: 1-3 and 2-4 without contours, 3-4 with contours.

dark-dark correlation at the left when they appear as RBE and at right when they appear as RRE, with each type contributing no-correlation samples for the opposite diagnostic. For these samples the green contours should show no spatial overlap, which can be checked with the second-row panel blinker in the online material.

Another contribution to the green selections comes from dynamic fibrils that are treated further in Sect. 4. They occur mostly in the top third of the field where some contours show overlap between columns. There are more non-overlap type II spicule contours in the lower part of the field, but they generally occupy the same parts of the field of view, which suggests that $\mathrm{RBE}$ and RRE launch along similar trajectories from common origins while they upset the dark-dark correlation for each other. The RRE discovery publication of Sekse et al. (2013a) indeed reported that many RREs are seen closely parallel to or touching RBEs or transiting from one to the other.

The scatter triangle at the right reaches less deeply than the one at the left, implying that the darkest RREs are less dark than the darkest RBEs while they still have as dark subsequent 

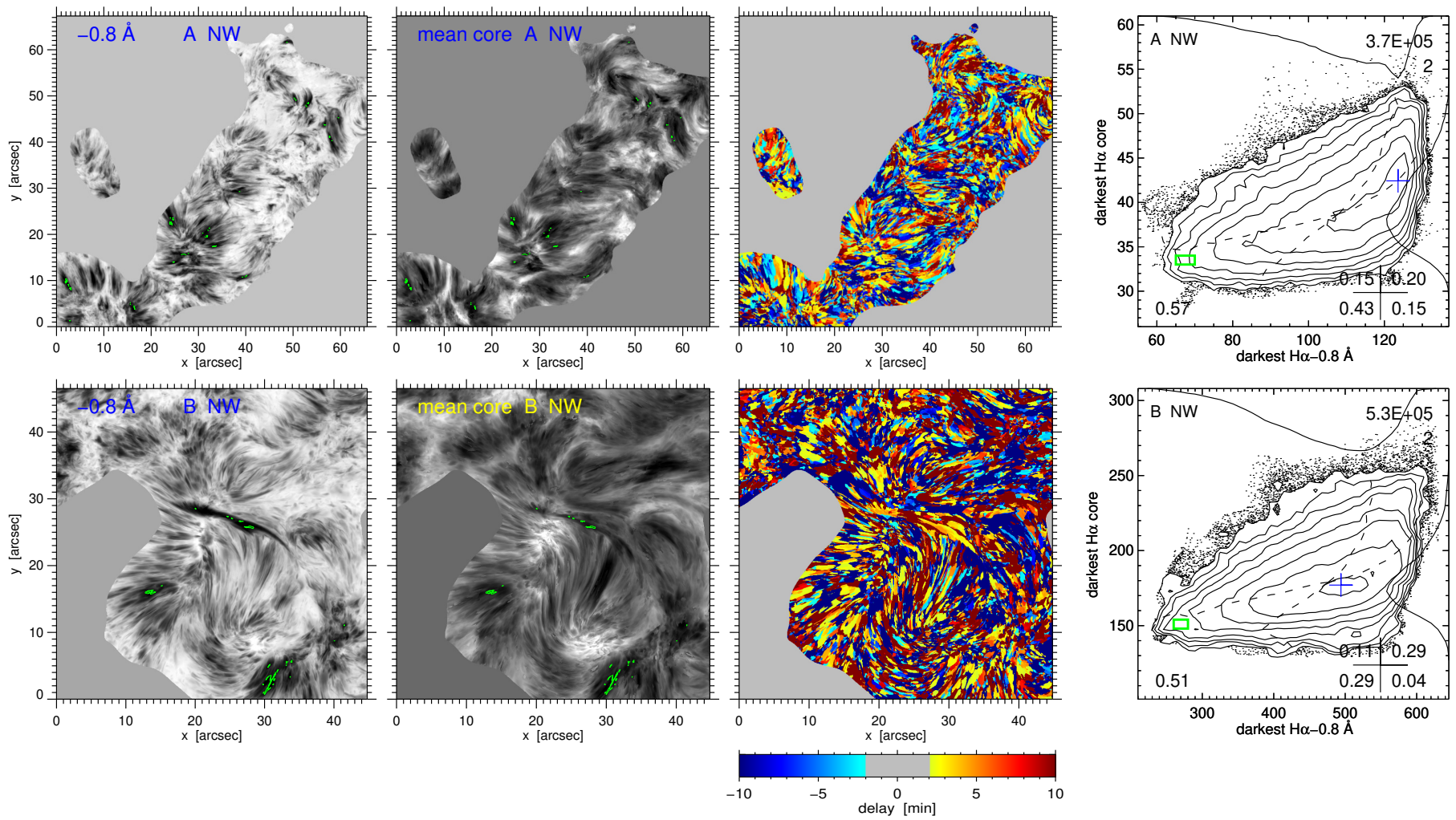

Fig. 12. Sequence-wide dark-dark comparisons for data A (upper row) and data B (lower row) network only. First column: darkest value per network pixel during the whole sequence at $\Delta \lambda=-0.8 \AA$. The lower image is the same as the third image in Fig. 9, except for the mask and contours. Second column: darkest value per network pixel during the whole sequence of core intensities averaged over $\Delta \lambda=[-0.4,+0.4] \AA ̊$ beyond $[-2,+2]$ min delay range around the blue-wing sampling per pixel. Third column: color-coded time delay between darkest wing occurrence and darkest core occurrence per pixel, yellow to red for increasing positive delay (core after wing) beyond 2 min, and light to dark blue for increasing negative delay (core before wing) beyond 2 min. Fourth column: scatter diagrams between the first and second images per row. The small green boxes and corresponding contours select darkest-darkest pairs of which the $\mathrm{H} \alpha$ profiles are shown in Fig. 13. Online image blinkers: 1-2, 1-3, $2-3,4-5.4-6,5-6$.

red-core fibrils. This is also seen in the two wing images. The tail of the distribution curve along the right-hand axis also drops more steeply for RREs, which confirms that there are fewer RREs than RBEs, as reported by Sekse et al. (2013a).

Figures 12-14: next we ask a broader question: how does the overall $\mathrm{H} \alpha$ core darkness relate to RBEs? So far, we correlated RBEs and core redshifts per pixel at specified delays of a few minutes, inspired by the Pub B PHE and its aftermath. Figure 12 instead compares darkest outer-wing instances per pixel with darkest core instances at any delay beyond two minutes, also negative. This inventory was made only for network in view of Fig. 10. Using the very darkest value sequence-wide implies selecting moments of good seeing for both the wing and core samples. We performed this for both data A and B because they differ appreciably in duration, seeing quality, and network extent and activity.

The leftmost images in Fig. 12 are again constructed by assigning the darkest value to each pixel that it reached in the blue wing during the entire sequence, in the same way as in Fig. 9, but only for network areas. The second-column images are similar constructs by selecting the darkest value per pixel in the $\mathrm{H} \alpha$ core, using the mean intensity over $\Delta \lambda=-0.4$ to $+0.4 \AA$ to reduce the Doppler sensitivity as in a spectroheliogram with $0.8 \AA$ bandpass. We excluded delays within the $[-2,+2]$ min range because Fig. 13 shows that many RBEs have dark cores that would contribute same-feature sampling with self-correlation. We avoided this contribution by not searching for the darkest core occurrence within two minutes before or after each pixel's darkest wing occurrence; this is longer than the RBE lifetimes.

The darkest-core images also show fibrilar morphology, with remarkable correspondence with the blue-wing images not only in overall morphology, but in many places also in detail in darker features, as is best seen with the panel blinkers in the online material. This is quantified by the significant dark-dark associations in the scatter diagrams at the right. Thus, selection of the darkest instant of a blue-wing pixel gives a good chance of its being part of a dark fibrilar feature made up by nearby darkest samplings, possibly at other times, and also of being part of a similar dark fibrilar feature in the line core made up by darkest samples each at some other time than its darkest-wing sampling.

The significant dark-dark association and the image similarities in Fig. 12 suggest that darkest RBEs and darkest core fibrils have considerable commonality while excluding simultaneity. We detail this further by analyzing the actual delays between each pair of samplings per pixel. They are shown in the colorcoded maps in the third column of Fig. 12. The many yelloworange pixels for data A ( $26 \%$ of all network pixels) marking 2-5 min delays imply that a quarter of the darkest RBE instances were followed by the darkest line-core instances within a few minutes. This suggests direct causal relationship. For data B this filling factor is smaller (19\%), implying a higher probability that the darkest core instance occurred earlier or later and was not a direct result of the sampled RBE. The duration of the data $\mathrm{B}$ 

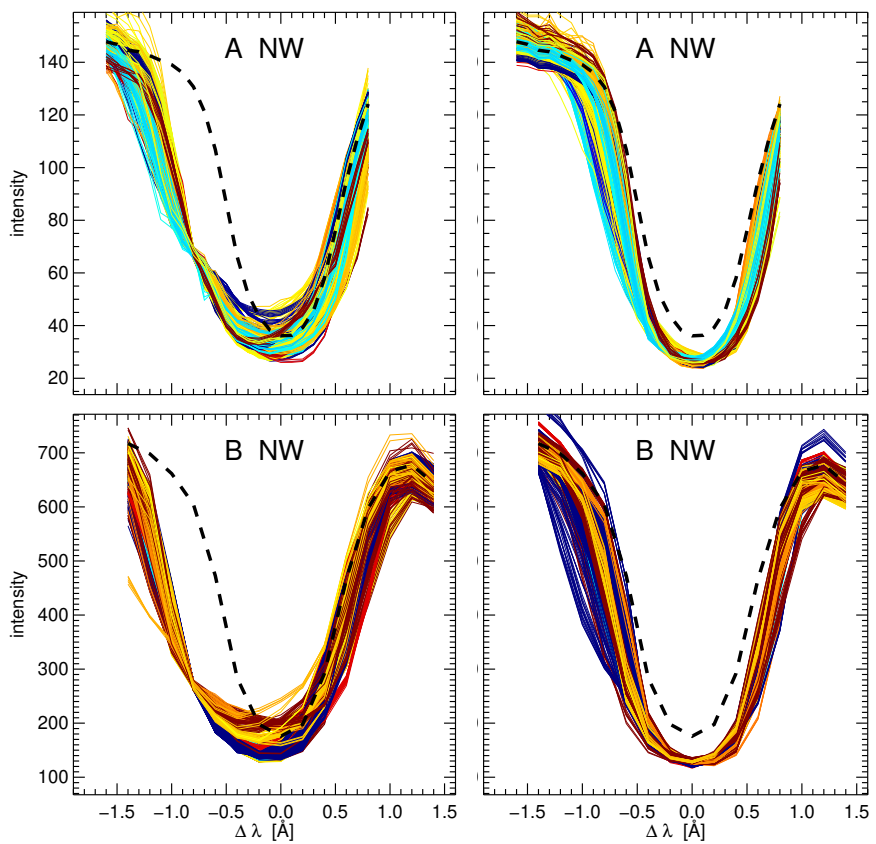

Fig. 13. $\mathrm{H} \alpha$ profiles for all pixels selected with the small green boxes in the scatter diagrams of Fig. 12 for data A (upper row) and data B (lower row). Dashed black curve: average profile over the field of view and the sequence duration. First column: profiles at the time of each darkest blue-wing sampling. Second column: profiles at the time of each darkest core sampling. Color coding: time delay between these samples on the scale of Fig. 12. The intensity units are the same as for the scatter diagrams in Fig. 12. data A: 402 pixels with $60 \%$ within the yellow-orange $2-5$ min positive-delay range. data $\mathrm{B}$ : 635 pixels with $26 \%$ within $2-5$ min delay.

sequence was three times longer and its field more active in producing RBEs, RREs, and also the dynamic fibrils discussed in Sect. 5, so that the chance that some other darkest-core pixel would win from the darkest within $2-5$ min delay is higher.

The yellow-orange pixels have contributed to the dark-dark associations of the previous figures, with the additional property that for each diagnostic, its darkening reached the very darkest value for that pixel during the whole sequence. They not only chart where dark fibrils follow on RBEs, but map the darkest of both. Their aggregations also show much fibrilar morphology, with good correspondence with the dark features defined by darkest samplings in the images to the left.

The Pub B contrail fibril shows a yellow-to-red retraction signature; more such fibrilar yellow-red alignments can be seen elsewhere, but with much confusion.

Figure 13 shows the $\mathrm{H} \alpha$ profiles at both sample moments for the very darkest-darkest pixels selected by the small green boxes in the scatter diagrams in Fig. 12. In the left-hand panels all curves cross close together for $\Delta \lambda=-0.8 \AA$ because the width of the selection boxes is set small to limit the number of profiles. The right-hand panels do not show such crossing points because the averaging over core wavelengths accepts different profile shapes. All these darkest-darkest profiles similarly represent RBE blue-wing darkening from combined blueshift and widening at the left and more symmetric dark-core signature at the right.

The yellow-orange profiles with positive [2-5] min delay (241 for data A, 162 for data B) are mostly similar. The blue negative-delay profiles show more blue-wing spread, light blue in the first panel, and dark blue in the last panel. The latter may
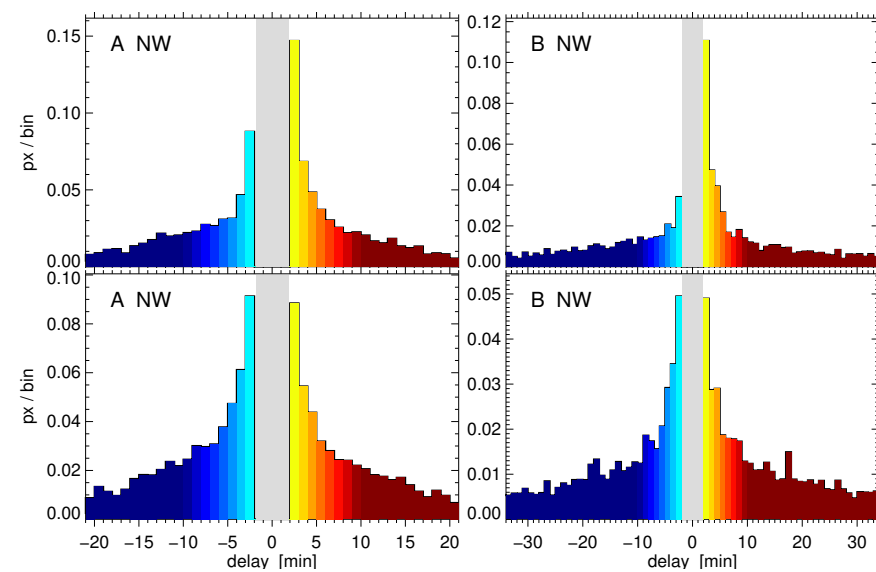

Fig. 14. Histograms of sampling delays for data A (left) and data B (right). The latter cover a twice longer delay range than shown here. Upper row: delays corresponding to the image pairs in Fig. 12, positive for darkest line-core occurrence after darkest blue-wing occurrence per network pixel, and negative for darkest core before darkest wing occurrence. Lower row: delays between the darkest blue-wing occurrence and the second-darkest blue-wing occurrence per network pixel. The delays are color-coded as in Fig. 12. The gray rectangles mark the $[-2,+2]$ min ranges without delay determinations. The bin widths are 1 min. The $y$-axes specify numbers of pixels per bin as fraction of the network area.

include some RBEs in this darkest-core selection because some RBEs also have very dark cores.

Figure 14 analyzes the delays in Fig. 12 in terms of occurrence histograms in its upper-row graphs. They are similar for Data A and B. The histograms show systematic patterns that differ strongly from a horizontal distribution for no association. Both panels show a peak to the right of the gap that implies a high probability that the core reached its darkest value a few minutes after the darkest RBE. For data B, the peak reaches slightly lower filling fraction because the sequence lasted longer and there were more competing features. Summing the 2-5 min positive-delay bins gives the $26 \%$ and $19 \%$ yellow-orange fill fractions in the delay maps in Fig. 12.

The peaks left of the gaps correspond to the light-blue pixels in Fig. 12 and are still considerable compared to the outer tails. For these pixels the darkest RBE instance was not followed by a darkest core instance within a few minutes, but they had their darkest core moment already just before the darkest RBE.

Farther away from the gaps, beyond six minutes, the decays reach symmetry between positive and negative values. They imply that outside the $[-2,+2]$ min range the core tends to be darkest preferentially at shorter time difference from the darkest wing sampling, and that beyond six-minute difference the latter may have occurred later or earlier with equal diminishing probability. This suggests recurrence, with the darkest instances occurring close after each other in temporal groups.

The histograms in the second row of Fig. 14 support this suggestion. For these the second darkest sampling is not one of the line core, but again of the blue wing at $\Delta \lambda=-0.8 \AA$, also beyond the $[-2,+2]$ min exclusion period. Its delay is a measure of $\mathrm{RBE}$ repetitions and the time interval between their darkest instances. Both histograms show symmetric peaks adjacent to the gaps and steep decays beyond, suggesting that darkest RBE instances repeat close in time. Beyond $\pm 7 \mathrm{~min}$, the decay tails resemble those in the upper panels.

Thus, the very darkest RBEs tend to appear in close succession, and have a good chance to produce maximum core 

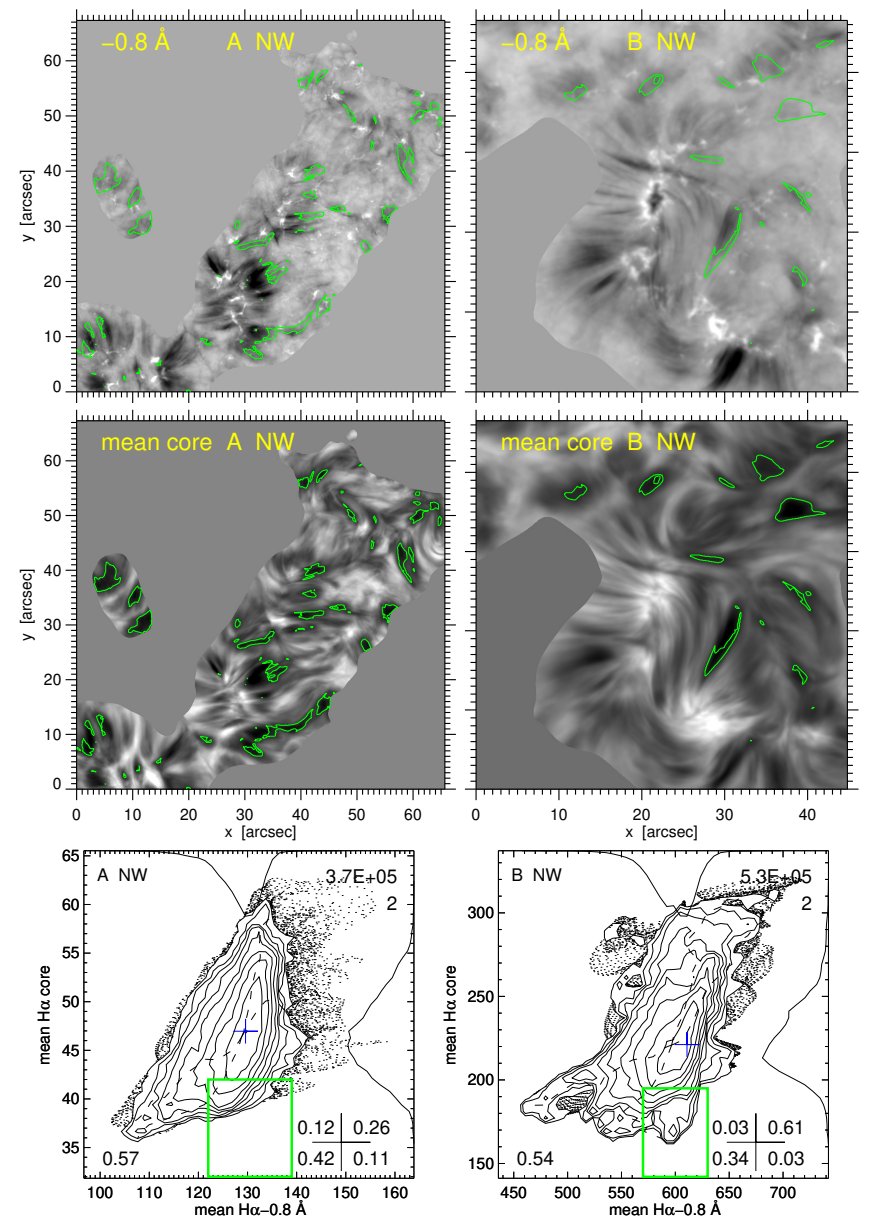

Fig. 15. Temporal averages and scatter analysis for the network areas in data $\mathrm{A}$ (left) and data B (right). First row: mean images at $\Delta \lambda=$ $-0.8 \AA$. Second row: mean images summing the $\mathrm{H} \alpha$ core over $\Delta \lambda=$ $[-0.4,+0.4] \AA$. Third row: corresponding scatter diagrams. The poorseeing moments are discarded, as specified in Fig. 2. The green boxes select pixels with average wing darkening but large core darkening. Online image blinkers: $1-3,2-4$.

darkening singly or in tandem. The repeat time indicated by the peaks in the lower histogram is $3-5 \mathrm{~min}$. This is longer than the mean RBE recurrence time of $84 \mathrm{~s}$ reported by Sekse et al. (2013b) because $[-2,+2]$ min simultaneity is excluded. With repeats faster than the typical persistence duration of subsequent core darkenings (Figs. 4 and 8), it may well be that darkest-core instances result from summing aftermaths of multiple preceding type II spicules. Cases $e-g$ in Fig. 3 seem examples of such a buildup.

Together, these histograms show that the darkest line-core instance of any network pixel most probably followed within a few minutes after the darkest RBE instance of that pixel. The next highest probability is that it followed on a previous next darkest RBE instance a few minutes earlier. When we add these second-best pairs by summing over the $[-5,-2]$ and $[+2,+5]$ min delay ranges, the filling fractions for darkest cores that are directly, likely causally, associated with darkest RBEs increase to $43 \%$ of all network pixels for data A and to $27 \%$ for data B.

Figure 15 compares temporal means over the entire image sequences except for poor-seeing moments.

In this averaging specific pixel-intensity combinations build up signature when they are persistent or repeat in place, not when they appear briefly only once or appear at a random location per image. The short-lived Pub B PHE, contrail, and aftermath darkenings that are so conspicuous in Fig. 1 do not leave much signature in this temporal averaging. Because the pore is the only long-lived dark feature in these data, the other dark streaks and blobs in these time-averaged images most probably represent recurrent features. The many darkest-wing darkest-core associations found in Figs. 12 and 14 also add up only when they repeatedly occur in place, with RBEs occurring recurrently and sequentially boosting line-core darkness.

The diagram for data $\mathrm{A}$ at the left shows a tilt of the higher contours from the summit pointing to and ending in the green box, whereas the darkest-darkest contour spur reaches farther left. Its tip shows the tightest darkest-darkest association of all scatter diagrams so far (note the convergence of the moment curves), describing locations where darkest time-averaged RBE presence goes together with darkest time-averaged core fibril presence. The corresponding image blinker in the online material confirms the high degree of correspondence between the darkest features.

The green box instead selects pixels that sample dark core averages with only slight or no averaged outer-wing darkening. The corresponding contours in the data A images above suggest that many of these lie at the tips of RBEs. They may statistically mark sites where aftermath core darkening started beyond the visible RBE extent, as was the case for Pub B contrail B, of which the longer post-RBE contrail extent is evident in Fig. 1.

In data B the similarly selected pixels come partly from the large and darkest summed-core streak below and to the right of the pore at image center. It is incompletely covered by the corresponding summed-RBE feature, similarly to the non-covered tips at left. However, other contributions come from dark core blobs in the upper third of the network area that are without clear RBE counterparts, too round to be RREs, and are identified as dynamic fibrils below. Together with RREs, they contribute to the conspicuous sag in the outer contours contained in the selection box.

The higher contours of the scatter diagrams agree between the two data sets. They show considerable dark-dark association up to the mountain summits and also considerable bright-bright association above them. The corresponding bright patches lie above network, whereas the darkest RBE and core features lie around that and point away from it.

Figure 16: the final figure shows how the sequence-average scatter correlations in Fig. 15 build up over time. For reference, all diagrams have green boxes with the same pixel-pair value ranges as in Fig. 15.

The construction and format resemble Fig. 8, but here the core intensity is sampled as in a spectroheliogram, as in Figs. 12 and 15 , only the network areas contribute, and instead of imposing temporal delays between the wing and core samples, temporal boxcar averaging is applied to both over increasing duration. The pixel-pair numbers are much higher, which produces smoother contours than for Fig. 15 because per panel, each instant contributes for which the boxcar duration around it fits within the sequence duration. Features with specific pixel-value combinations that are long lived or appear recurrently in place so gain much weight. As in Fig. 5, the data B pore is the only longlived dark feature in our data; SHOWEX inspection shows that it produced the slowly developing left-pointing spur that becomes a promontory below the boxcar duration label in the last panel.

The diagrams in the first column (no temporal averaging) are closely similar to the second-column panels in Fig. 8, they differ only in their core wavelength sampling and in the full-field/ 

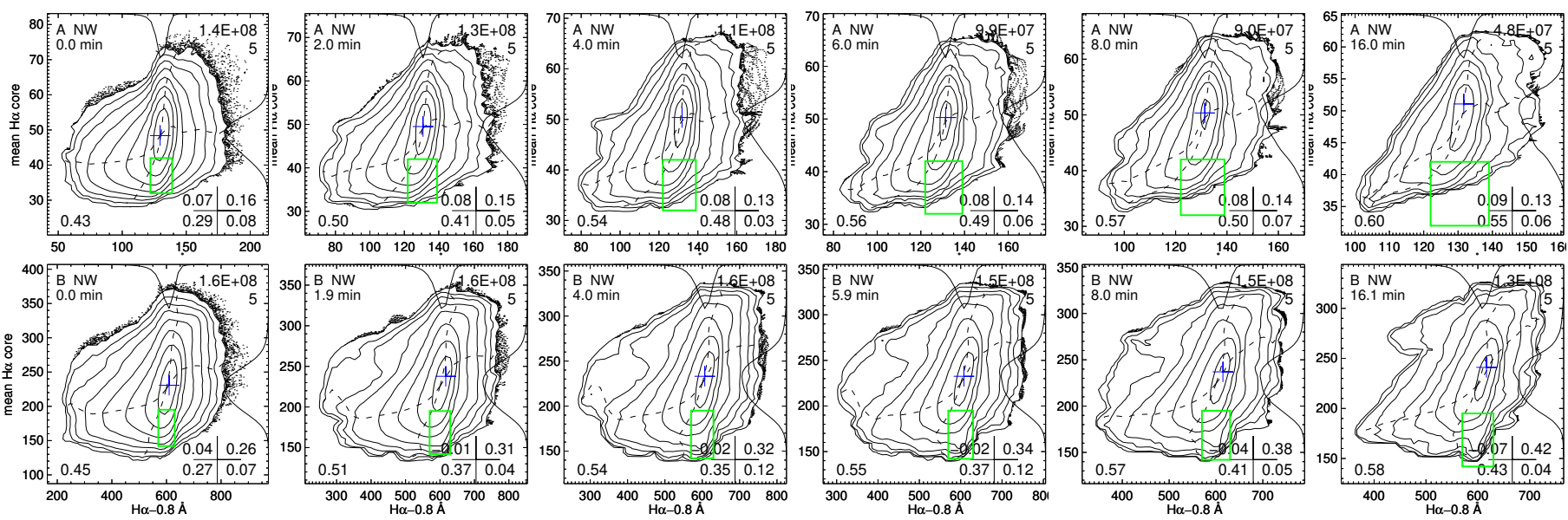

Fig. 16. Full-sequence scatter analysis for data A (upper row) and data B lower row). Format as in Fig. 8, but only for the network areas and with temporal boxcar averaging over the duration specified at the top left in each panel. The poor-seeing moments are discarded, as specified in Fig. 2. Abscissas: $\mathrm{H} \alpha$ wing intensity at $\Delta \lambda=-0.8 \AA$. Ordinates: $\mathrm{H} \alpha$ core intensity averaged over $\Delta \lambda=[-0.4,+0.4] \AA$. The axes shrink along rows because momentary extremes lose weight. The green boxes outline the same ranges as in Fig. 15.

network difference. The 0 min panels in Fig. 16 show larger dark-dark association from sampling the mean core, which can be dark in RBEs (first column of Fig. 13). This sameinstant association contributes to the dark-dark correspondences in Fig. 15, but was excluded in Figs. 12 and 14.

Along the rows in Fig. 16, the scatter mountains gradually transform from resembling the 0 min mountains in Fig. 8 toward resembling those in Fig. 15. With increasing boxcar duration, the darkest-darkest associations tighten. The findings in Figs. 12 and 14 suggest that this association is dominated at 4 and $6 \mathrm{~min}$ boxcar averaging by dark core fibrils directly following on RBEs and that the association tightening at longer averaging is contributed by RBE-plus-core-darkening recurrence at the same locations.

The slowly developing contour bulge in the green boxes in the lower row is attributed to dynamic fibrils in the next section. data $\mathrm{A}$ in the upper row had fewer of these and shows the tightest dark-dark mountain spur of all our scatter diagrams in the rightmost diagram, quantified by the lower-left quadrant Pearson coefficient 0.55 . It resembles the opposite bright-bright extension in the last panel of Fig. 5 in displaying a high degree of association. For example, all RBE pixels darker than 120 data units have darker-than-average cores in these 16 min boxcar summations, with high darkest-darkest correspondence. The corresponding contours extend fairly symmetrically along the full-correlation diagonal so that the darkest pixels in the one are also darkest in the other at similar densities.

\section{Interpretation}

Confusion from close recurrence. Figure 3 and its online movie versions illustrate the great difficulty in direct one-toone recognition of RBE outer-wing darkening followed by core darkening as in the Pub B PHE-contrail-aftermath pattern, but occurring more ubiquitously and at smaller scale. We attribute this difficulty not only to the very small and seeing-sensitive scales, but also to the dynamical confusion caused by frequent type II spicule recurrence, both cospatial and nearby, in the $\mathrm{H} \alpha$ scenes around network. The RBE and RRE occurrence charts in Rouppe van der Voort et al. (2009), Sekse et al. (2012, 2013a), the finding of fast recurrence in Sekse et al. (2013b), and the $x-t$ time-line charts in Yurchyshyn et al. (2013) indeed suggest frequent repetitions in which new RBEs and RREs closely follow tracks of previous ones or similar ones nearby. Moreover, the Pub B event had an adjacent similar feature (contrail B in Pub B) as well as a subsequent one (contrail C in Pub B). The initial dark blobs in Fig. 4, the delay histograms in Fig. 14, and the time-averaged RBE and fibrilar darkening in Fig. 15 and their build-up in Fig. 16 also suggest frequent recurrence.

Dynamic fibrils. These were first described by Hansteen et al. (2006) and De Pontieu et al. (2007b) and also occurred in our observations, especially in data B. They show up dark at line center but darken the outer wings only slightly, so that they do not contribute dark-dark correlation to our time-delay scatter diagrams. Inspection per SHOWEX showed that the roundish dark blobs in the upper third part of the fourth image of Fig. 15, some with greencontoured centers, correspond to patches of recurrent dynamic fibrils, for example, the blob harboring the leftmost contour and the blob-with-contour to its right, both below the label. Persistent repetitions at the same location build up their time-average core darkness in this image, with only a slight summed outer-wing darkening in the second image of Fig. 15.

Dynamic fibrils also contributed green contours in Fig. 11 by being dark at line center without prior darkening in either wing. These contours are likely to overlap, in contrast to RBEs versus RREs, as indeed seen in the upper third of the first-row images in Fig. 11.

With this identification, these dynamic-fibril patches can also be recognized as showing a mottled short-streak morphology in the third image of Fig. 9 and the companion first lowerrow image of Fig. 12. This morphology implies that only short dynamic-fibril segments become darkest pixels over time.

There were fewer dynamic fibrils in the field of data A. In the lower row of Fig. 16, the greater contribution by dynamic fibrils and RREs is recognized as distinct sags in the bottom contours within the green selection box; it builds up with integration duration through recurrence. Without this contribution and the pore, the final diagram would show cleaner darkest-darkest association, as in the upper row.

Incomplete overlaps. There are multiple reasons why our statistical correlations cannot reach one-to-one purity. The first is the mutual exclusion of RBEs and RREs that is illustrated in the second row of Fig. 11, where many of their locations appear bright in the other wing panel. A second is the presence of dynamic fibrils, 
which also contribute to the green pixel selections in Fig. 11. A third is the lateral motion of type II spicule features that upsets time-delayed correlations per pixel. Sekse et al. (2012, 2013b) reported transverse speeds for RBEs of $5-10 \mathrm{~km} \mathrm{~s}^{-1}$ that may give sizable offsets between cause and time-delayed effect.

Furthermore, RBEs become conspicuous in the outer blue $\mathrm{H} \alpha$ wing only at appreciable distance from their launching sites in the network, whereas the return flows retract closer to these roots. Their corresponding blue-wing and red-core darkening therefore tend to lie adjacent along the type II spicule track rather than cospatial per pixel. This is demonstrated by the leftward extent, left of the plus, of the dark clouds in the bottom row of Fig. 4, and also by the first and third good-seeing columns of Fig. 7 and the second and fourth columns of Fig. 9, where many box-selected dark features extend closer to network in the delayed line-core images. In addition to these missing RBE feet in overlap correlations, there are also missing RBE tips, as suggested by Fig. 15.

\section{Discussion}

Do type II spicules cause long network fibrils? The statistical approach of Figs. 4 and 8-11 brings evidence of return flows and fibrilar darkening after RBEs in the form of significant darkdark associations for multiple-minute delays. They are far from one-to-one, but this is to be expected from mutual RBE and RRE exclusion, presence of dynamic fibrils, missing overlaps of feet and tips along type II spicule tracks, and confusion from fast recurrences. The remaining dark-dark associations in the timedelay diagrams in Figs. 7-12 are highly significant, showing up as prominent left-down-sloping mountain spurs in stark contrast to the absence of significant correlations in the simultaneous diagrams in Figs. 8 and 9. These association spurs imply that there is a strong tendency for $\mathrm{H} \alpha$ RBEs to produce subsequent dark $\mathrm{H} \alpha$ fibrils intermittently.

The tightest dark-dark associations are in Figs. 15-16 and are tighter than those in Fig. 8. They imply that locations that become darkest in time-averaged $\mathrm{H} \alpha$ blue-wing from sampling repeated RBEs also tend to be darkest in the time-averaged $\mathrm{H} \alpha$ line core. Frequent RBE recurrence was established by Sekse et al. (2013b) and is also diagnosed in Figs. 4, 12 and 14.

Whether all $\mathrm{H} \alpha$ fibrils result from small-scale dynamic heating events such as those producing RBEs and RREs remains an open question, but our evidence leads us to conclude that type II spicules do represent a major agent in the production of dark fibrils around network.

The disparate numbers of RBEs and dark fibrils around network in Fig. 1 appear contradictory, but because the half-minute RBE lifetimes (Sekse et al. 2013b) are much shorter than the multi-minute aftermaths in Figs. 8 and 14, such contrail fibrils should indeed be more numerous at any one time. The densities of RBEs and core fibrils in the images in Figs. 9-12 and 15 indeed agree much better.

$H \alpha$ and CaII $8542 \AA$ extinctions. $\mathrm{H} \alpha$ is the strongest solar line in the visible off-limb spectrum and so gave the chromosphere its name. On the disk it appears much weaker than Ca II K, only about as strong as Ca II $8542 \AA$, but the fibrilar scenes it shows are so extraordinary that $\mathrm{H} \alpha$ is the principal diagnostic of the chromosphere. We attribute this to its extraordinary atomic properties. First, hydrogen is superabundant. Second, the line has exceedingly high excitation energy $(10.2 \mathrm{eV})$. Third, it sits on top of the strongest line in the solar spectrum, Ly $\alpha$. Together, these factors define its extinction coefficient with high temperature sensitivity, including very large nonequilibrium sensitivity that the CaII lines do not have. We give a brief summary with key references here; more detail is given in Pub B and Rutten (2016, 2017a,b).

The Einstein relation for the ratio of collisional excitation and deexcitation rates in Ly $\alpha$ imparts very steep Boltzmann temperature sensitivity to the population of the H I $n=2$ level that defines the $\mathrm{H} \alpha$ extinction coefficient (Fig. 7 of Pub B) as well as its nonequilibrium sensitivity. In any feature that is sufficiently thick for Ly $\alpha$ to reach radiative detailed balance ( $\mathrm{H} \alpha$ fibrils certainly qualify because they are already optically thick in $\mathrm{H} \alpha$ ), collisional detailed balance in Ly $\alpha$ and LTE extinction of $\mathrm{H} \alpha$ are reached fast (seconds) at high temperature but slow (minutes) at low temperature (Fig. 7 of Carlsson \& Stein 2002). The effect of the latter slowness is commonly called "nonequilibrium hydrogen ionization", but the culprit is the large Ly $\alpha$ jump, whereas the ionization proceeds from the $n=2$ level in instantaneous statistical equilibrium, in a loop governed by photoionization in the Balmer continuum, and cascade recombination including photon losses in the Balmer lines (Fig. 3 of Carlsson \& Stein 2002). This loop imparts NLTE over- or underionization set by the difference between the local temperature and the $5300 \mathrm{~K}$ radiation temperature of the impinging Balmer continuum from the deep photosphere, but these NLTE departures are small with respect to the gigantic $n=2$ overpopulations from slow collisional settling in Ly $\alpha$ in gas that cools after having been hot.

The best demonstration so far is the extension to 2D MHD simulation by Leenaarts et al. (2007) of the fundamental 1D hydrodynamics simulation of Carlsson \& Stein (2002). The last panel of Fig. 1 of Leenaarts et al. (2007, movie version in the online material) shows 12 dex overpopulations of the $n=2$ level that define $\mathrm{H} \alpha$ extinction in the cool-down phases of acoustic shocks in the simulated internetwork and 8 dex overpopulations after passages of magnetically guided shocks in the simulated network that produce dynamic fibrils shown in Fig. 3 of Leenaarts et al. (2007). The next to last panel of their Fig. 1 shows 2-3 dex additional overionization by the Balmer continuum for the cool-down phases. Their Fig. 2 (unpublished online movie version) shows that hydrogen ionization reaches only $10 \%$ in the shocks, but this partial ionization already suffices to produce $8-12$ dex $\mathrm{H} \alpha$ overextinction in the post-shock cooling phases.

Dynamic fibrils and RBEs both represent PHEs, but the latter reach higher temperature and hydrogen ionization. This is obviously the case for tips of type II spicules, which frequently reach full ionization (De Pontieu et al. 2011; Henriques et al. 2016), but it also holds along on-disk RBEs. We show this by comparing their appearance in $\mathrm{H} \alpha$ and Ca II $8542 \AA$ assuming Saha-Boltzmann extinction. In hot features this assumption holds for both lines; it was indeed valid for $\mathrm{H} \alpha$ in the hot phases of the simulated internetwork shocks and dynamic fibrils of Carlsson \& Stein (2002) and Leenaarts et al. (2007).

Figure 7 of Pub B shows that the Saha-Boltzmann curves for the extinction of these lines cross over near $6400 \mathrm{~K}$ at a few percent hydrogen ionization. At lower temperatures the higher extinction of Ca II $8542 \AA$ explains that in this line RBEs start earlier and closer to their network roots than in $\mathrm{H} \alpha$ (Fig. 1 of Sekse et al. 2012 and Fig. 10 of Sekse et al. 2013b).

For higher temperature, $\mathrm{H} \alpha$ extinction increases steeply through its Boltzmann sensitivity to reach a maximum near $8000 \mathrm{~K}$ and $80 \%$ hydrogen ionization, whereas Ca II ionization causes steeply decreasing Ca II $8542 \AA$ extinction down to only $1 \%$ of the maximum $\mathrm{H} \alpha$ value. RBEs extend considerably farther in $\mathrm{H} \alpha$ than in $\mathrm{CaII} 8542 \AA$, as also shown in Fig. 1 of 
Sekse et al. (2012) and Fig. 10 of Sekse et al. (2013b). This additional extent implies temperatures above $7000 \mathrm{~K}$ and hydrogen ionization above $20 \%$ in these outer RBE parts (Fig. 7 of Pub B), below the near $100 \%$ value in the Pub B PHE and contrail that were not visible at all in Ca II $8542 \AA$ and above the $10 \%$ value in dynamic fibrils that extend equally far in the two lines (Fig. 3 of Skogsrud et al. 2016).

In subsequent cooling of this hot gas, $\mathrm{H} \alpha$ then initially retains its strong high-temperature extinction, which implies NLTE overopacities similar to the high values in the wakes of magnetoacoustic shocks found by Leenaarts et al. (2007). Return fibrils therefore maintain $\mathrm{H} \alpha$ darkness even when the gas cools below $6400 \mathrm{~K}$ and regains Ca II $8542 \AA$ presence, as was the case in the Pub B aftermath at the very end of the redshifted retraction phase (Fig. 5 of Pub B).

$\mathrm{H} \alpha$ and $L y \alpha$ scattering. The $\mathrm{H} \alpha$ source function, which together with the extinction defines $\mathrm{H} \alpha$ image formation, is easier to describe because it is dominated by resonance scattering, as for any strong chromospheric line. Thomas (1957) and Jefferies \& Thomas (1959) described it as special in being "photoelectrically controlled" by the Balmer ionization loop, but this contribution is only minor, while resonance scattering represents the main agent (Sect. 6 of Rutten \& Uitenbroek 2012). The actual abnormality of $\mathrm{H} \alpha$ is strong backscattering from the chromosphere to the low photosphere over the very deep $\mathrm{H} \alpha$ opacity gap in the upper photosphere (Schoolman 1972), which produces a $\mathrm{H} \alpha$ source function raise there (Fig. 7 of Rutten \& Uitenbroek 2012) that was misinterpreted with the famous Fig. 3 of Jefferies \& Thomas (1959).

The scattering nature of $\mathrm{H} \alpha$ implies that features that are more opaque become darker: $\mathrm{H} \alpha$ fibril darkness is defined by the local extinction, not by the local temperature. The same holds for Ca II $8542 \AA$, which scatters very similarly. For H $\alpha$ nonequilibrium extinction with retarded decay from preceding large hightemperature values enhances fibril darkening.

Resonance scattering also increases the apparent size of observed features. The PHEs at the root of the RBE phenomenon are due to plasma processes that possibly operate on physical scales below telescopic resolution limits and heat gas along very thin tracks initially. Ly $\alpha$ surround-scattering into cool gas around these smears the corresponding $\mathrm{H} \alpha$ opacity feature over tens of kilometers (Fig. 3 of Rutten 2016), while $\mathrm{H} \alpha$ scattering into cool surrounding gas smears its source function signature over hundreds of kilometers. These scattering diffusions together may contribute to RBE visibility at the SST resolution.

The thin bright RBE stripes in the third column of Fig. 3 appear narrower because they mainly represent core Dopplershifts, which are primarily contributed by the last $\mathrm{H} \alpha$ photon scatterings on their way out and are widened primarily by Ly $\alpha$ surround-scattering, which smoothes their opacity. Their observed thinness suggests that the PHEs that underlie RBE formation are intrinsically narrower than the observed blue-wing RBEs.

Inadequate resolution. The long reach of the mountain spur of dark-dark association in the scatter diagrams up to slightly dark - slightly dark association close to the mountain summits is intriguing. Figure 10 was made to test whether these upper spur parts come from quiet internetwork areas. Such areas are not often seen in $\mathrm{H} \alpha$ images because most internetwork is hidden under overlying canopies of $\mathrm{H} \alpha$ fibrils. The only study addressing one at SST resolution so far is the one by Rouppe van der Voort et al. (2007), who attributed the extremely dynamic very fine mottles they observed with the SST to shocks.
Internetwork shocks are well known and well understood in their manifestation as Ca II $\mathrm{k}_{2 V}$ and $\mathrm{H}_{2 V}$ grains (Rutten \& Uitenbroek 1991; Carlsson \& Stein 1997), and because these shocks were also well demonstrated to act as nonequilibrium $\mathrm{H} \alpha$ opacity enrichers by Carlsson \& Stein (2002) and Leenaarts et al. (2007), we expect to find a delay signature also from these in our scatter diagrams.

The internetwork scatter diagram at the left in Fig. 10 does show some dark-dark association, but far less so than the network diagram at the right. Figure 10 therefore indicates that also the slightly dark - slightly dark associations along the upper spur parts, including the first-moment crossing, come from the network areas and are likely also due to magnetic processes. This suggests that many or most of such magnetodynamical heating features are not resolved even at the SST, resulting in summit-ward migration along the spur, as shown for poor seeing in Fig. 7. This lack of resolution is also suggested by the very fine striations of the larger dark fibril bunches in Fig. 3 and its movie versions, which become indistinguishable at poor-seeing moments. Sekse et al. (2012) also suggested that their highquality SST observations were not resolving all actual RBEs.

Intermittent hydrogen ionization. Our results, in particular the tight dark-dark associations in Figs. 15 and 16, are testimony of intermittent heating including partial hydrogen ionization around our quiet-Sun network areas. The RBE blue-wing darkenings directly portray such heating events. Their H $\alpha$ opacity stems from the steep increase of Ly $\alpha$ excitation with temperature and the accompanying increase in $\mathrm{H} \alpha$ extinction and hydrogen ionization which has the same content but is clearer. They are short lived but occur frequently and recurrently, and so build up their time-averaged darkness. The $\mathrm{H} \alpha$ core darkenings at the same locations represent lower temperatures but with high opacities, hence low intensities, that are leftovers of preceding heating events. Without prior heating they would not have such a high $\mathrm{H} \alpha$ opacity.

Thus, the $\mathrm{H} \alpha$ chromosphere should be seen as the solar atmosphere domain where hydrogen ionizes intermittently, doing so frequently and repetitively on very small spatial and short timescales imposed by magnetodynamical processes. In classical 1D static standard models such as ALC7 of Avrett \& Loeser (2008), the chromosphere is a plane-parallel layer in which the degree of hydrogen ionization increases gradually from fully neutral at the bottom to fully ionized at the top, at infinite horizontal extent and eternal constancy. Our interpretation instead makes the network chromosphere intrinsically 3D structured and time-dependent, intermittently heated, and achieving hydrogen ionization by magnetic agents operating on very small spatial and very short temporal scales.

\section{Conclusion}

We have collected evidence that long network-surrounding $\mathrm{H} \alpha$ fibrils often follow on the launch of propagating heating events that are observed as rapid blue and red excursions and represent on-disk manifestations of type II spicules. We suggest that partial hydrogen ionization is a principal agent in defining the subsequent fibril visibility along the tracks of these events. The evidence further suggests that many of these heating events are not yet resolved by the currently best solar telescope.

Our suggestion is that the $\mathrm{H} \alpha$ chromosphere is an extremely dynamic solar-atmosphere domain where hydrogen frequently ionizes at least partially on very small scales due to magnetic dynamism (even in quiet-Sun areas) and that this intermittent heating causes the extraordinarily rich fibrilar appearance of 
the $\mathrm{H} \alpha$ chromosphere. The moral is that in modeling $\mathrm{H} \alpha$ network fibrils we may no longer assume statistical equilibrium, but instead must be concerned with the local gas history, including what happened nearby in Ly $\alpha$. This may also hold for other features that are seen particularly well in $\mathrm{H} \alpha$, such as Ellerman bombs, surges, filaments, and prominences, and it may well hold for all chromosphere modeling because hydrogen ionization is its dominant entity.

Progress may come from both observations and simulations. The Daniel K. Inouye Solar Telescope promises higher resolution in $\mathrm{H} \alpha$ than the Swedish 1-m Solar Telescope. When full-baseline solar imaging is developed at the Atacama Large Millimeter/submillimeter Array, this may become the champion because the millimeter continua gain nonequilibrium opacity in dynamic heating and cooling in the same manner as $\mathrm{H} \alpha$ (Rutten 2017b).

In numerical simulations progress will come from including more small-scale heating processes such as ion-neutral interactions implemented in Bifrost by Martínez-Sykora et al. (2018) and also accounting for nonequilibrium hydrogen partitioning. The subsequent $\mathrm{H} \alpha$ synthesis must be similarly time-dependent and 3D, which already poses a great challenge (Pereira 2019), but additionally implement nonequilibrium $\mathrm{H} \alpha$ opacity evaluation.

We look forward to such developments.

Acknowledgements. We thank Tiago Pereira for help during the data B observations. The SST is operated on the island of La Palma by the Institute for Solar Physics of Stockholm University in the Spanish Observatorio del Roque de los Muchachos of the Instituto de Astrofísica de Canarias. The Institute for Solar Physics is supported by a grant for research infrastructures of national importance from the Swedish Research Council (registration number 2017-00625). This research is supported by the Research Council of Norway, project number 250810, and through its Centres of Excellence scheme, project number 262622. We made much use of the SolarSoft and ADS libraries.

\section{References}

Antolin, P., Schmit, D., Pereira, T. M. D., De Pontieu, B., \& De Moortel, I. 2018, ApJ, 856, 44

Avrett, E. H., \& Loeser, R. 2008, ApJS, 175, 229

Bray, R. J., \& Loughhead, R. E. 1974, The Solar Chromosphere (London: Chapman and Hall)

Carlsson, M., \& Stein, R. F. 1997, ApJ, 481, 500

Carlsson, M., \& Stein, R. F. 2002, ApJ, 572, 626

Carlsson, M., Hansteen, V. H., Gudiksen, B. V., Leenaarts, J., \& De Pontieu, B. 2016, A\&A, 585, A4
Chintzoglou, G., De Pontieu, B., Martínez-Sykora, J., et al. 2018, ApJ, 857, 73 De Pontieu, B., McIntosh, S., Hansteen, V. H., et al. 2007a, PASJ, 59, S655

De Pontieu, B., Hansteen, V. H., Rouppe van der Voort, L., van Noort, M., \& Carlsson, M. 2007b, ApJ, 655, 624

De Pontieu, B., McIntosh, S. W., Carlsson, M., et al. 2011, Science, 331, 55

De Pontieu, B., De Moortel, I., Martínez-Sykora, J., \& McIntosh, S. W. 2017, ApJ, 845, L18

Hansteen, V. H., De Pontieu, B., Rouppe van der Voort, L., van Noort, M., \& Carlsson, M. 2006, ApJ, 647, L73

Henriques, V. M. J., Kuridze, D., Mathioudakis, M., \& Keenan, F. P. 2016, ApJ, 820,124

Jafarzadeh, S., Rutten, R. J., Solanki, S. K., et al. 2017, ApJS, 229, 11

Jefferies, J. T., \& Thomas, R. N. 1959, ApJ, 129, 401

Langangen, Ø., De Pontieu, B., Carlsson, M., et al. 2008, ApJ, 679, L167

Leenaarts, J., Rutten, R. J., Sütterlin, P., Carlsson, M., \& Uitenbroek, H. 2006, A\&A, 449, 1209

Leenaarts, J., Carlsson, M., Hansteen, V., \& Rutten, R. J. 2007, A\&A, 473, 625

Leenaarts, J., Rutten, R. J., Reardon, K., Carlsson, M., \& Hansteen, V. 2010, ApJ, 709, 1362

Leenaarts, J., Carlsson, M., \& Rouppe van der Voort, L. 2012, ApJ, 749, 136

Leenaarts, J., Carlsson, M., \& Rouppe van der Voort, L. 2015, ApJ, 802, 136

Lockyer, J. N. 1868, Proc. R. Soc. London Ser. I, 17, 131

Martínez-Sykora, J., De Pontieu, B., De Moortel, I., Hansteen, V. H., \& Carlsson, M. 2018, ApJ, 860, 116

Pereira, T. M. D. 2019, Adv. Space Res., 63, 1434

Pietarila, A., Hirzberger, J., Zakharov, V., \& Solanki, S. K. 2009, A\&A, 502, 647

Rouppe van der Voort, L. H. M., De Pontieu, B., Hansteen, V. H., Carlsson, M., \& van Noort, M. 2007, ApJ, 660, L169

Rouppe van der Voort, L., Leenaarts, J., De Pontieu, B., Carlsson, M., \& Vissers, G. 2009, ApJ, 705, 272

Rutten, R. J. 2006, in Solar MHD Theory and Observations: A High Spatial Resolution Perspective, eds. J. Leibacher, R. F. Stein, \& H. Uitenbroek, ASP Conf. Ser., 354, 276

Rutten, R. J. 2016, A\&A, 590, A124

Rutten, R. J. 2017a, in IAU Symposium, eds. S. Vargas Domínguez, A. G. Kosovichev, P. Antolin, \& L. Harra, 327, 1

Rutten, R. J. 2017b, A\&A, 598, A89

Rutten, R. J., \& Rouppe van der Voort, L. H. M. 2017, A\&A, 597, A138

Rutten, R. J., \& Uitenbroek, H. 1991, Sol. Phys., 134, 15

Rutten, R. J., \& Uitenbroek, H. 2012, A\&A, 540, A86

Scharmer, G. B., Bjelksjo, K., Korhonen, T. K., Lindberg, B., \& Petterson, B. 2003, in Innovative Telescopes and Instrumentation for Solar Astrophysics, eds. S. L. Keil, \& S. V. Avakyan, Proc. SPIE, 4853, 341

Scharmer, G. B., Narayan, G., Hillberg, T., et al. 2008, ApJ, 689, L69

Schoolman, S. A. 1972, Sol. Phys., 22, 344

Sekse, D. H., Rouppe van der Voort, L., \& De Pontieu, B. 2012, ApJ, 752, 108 Sekse, D. H., Rouppe van der Voort, L., De Pontieu, B., \& Scullion, E. 2013a, ApJ, 769, 44

Sekse, D. H., Rouppe van der Voort, L., \& De Pontieu, B. 2013b, ApJ, 764, 164 Skogsrud, H., Rouppe van der Voort, L., \& De Pontieu, B. 2016, ApJ, 817, 124 Strous, L. H. 1994, PhD Thesis, Utrecht University, The Netherlands

Thomas, R. N. 1957, ApJ, 125, 260

Vissers, G., \& Rouppe van der Voort, L. 2012, ApJ, 750, 22

Yurchyshyn, V., Abramenko, V., \& Goode, P. 2013, ApJ, 767, 17 